\title{
Search for doubly charmed baryons and study of charmed strange baryons at Belle
}

Y. Kato, ${ }^{36}$ T. Iijima, ${ }^{37,36}$ I. Adachi, ${ }^{11}$ H. Aihara, ${ }^{62}$ D. M. Asner ${ }^{47}$ T. Aushev,${ }^{20}$ A. M. Bakich,${ }^{56}$ A. Bala,${ }^{48}$ Y. Ban, ${ }^{49}$ V. Bhardwaj, ${ }^{38}$ B. Bhuyan, ${ }^{14}$ A. Bobrov, ${ }^{3}$ G. Bonvicini ${ }^{68}$ A. Bozek, ${ }^{42}$ M. Bračko, ${ }^{31,21}$ T. E. Browder,${ }^{10}$ D. Červenkov, ${ }^{4}$ V. Chekelian, ${ }^{32}$ A. Chen,${ }^{39}$ B. G. Cheon, ${ }^{9}$ K. Chilikin, ${ }^{20}$ R. Chistov, ${ }^{20}$ K. Cho, ${ }^{24}$ V. Chobanova,${ }^{32}$ Y. Choi,${ }^{55}$ D. Cinabro, ${ }^{68}$ J. Dalseno, ${ }^{32,58}$ M. Danilov, ${ }^{20,34}$ Z. Doležal, ${ }^{4}$ Z. Drásal, ${ }^{4}$ A. Drutskoy, ${ }^{20,34}$ D. Dutta,${ }^{14}$ K. Dutta, ${ }^{14}$ S. Eidelman, ${ }^{3}$ H. Farhat,${ }^{68}$ J. E. Fast ${ }^{47}$ T. Ferber, ${ }^{6}$ V. Gaur,${ }^{57}$ N. Gabyshev, ${ }^{3}$ S. Ganguly, ${ }^{68}$ A. Garmash, ${ }^{3}$ R. Gillard ${ }^{68}{ }^{61}$ Y. M. Goh, ${ }^{9}$ B. Golob, ${ }^{29,21}$ J. Haba, ${ }^{11}$ K. Hayasaka, ${ }^{37}$ H. Hayashii, ${ }^{38}$ X. H. He ${ }^{49}$ Y. Horii, ${ }^{37}$ Y. Hoshi ${ }^{60}$ W.-S. Hou, ${ }^{41}$ Y. B. Hsiung, ${ }^{41}$ K. Inami, ${ }^{36}$ A. Ishikawa, ${ }^{61}$ Y. Iwasaki, ${ }^{11}$ T. Iwashita ${ }^{38}$ I. Jaegle, ${ }^{10}$ T. Julius, ${ }^{33}$ J. H. Kang, ${ }^{70}$ E. Kato ${ }^{61}$ T. Kawasaki,${ }^{44}$ C. Kiesling, ${ }^{32}$ D. Y. Kim, ${ }^{54}$ H. J. Kim, ${ }^{27}$ J. B. Kim, ${ }^{25}$ J. H. Kim, ${ }^{24}$ M. J. Kim, ${ }^{27}$ Y. J. Kim, ${ }^{24}$ J. Klucar, ${ }^{21}$ B. R. Ko, ${ }^{25}$ P. Kodyš ${ }^{4}$ S. Korpar, ${ }^{11,21}$ P. Krokovny, ${ }^{3}$ T. Kuhr, ${ }^{23}$ A. Kuzmin, ${ }^{3}$ Y.-J. Kwon, ${ }^{70}$ S.-H. Lee, ${ }^{25}$ J. Li ${ }^{53}$ Y. Li ${ }^{67}$ L. Li Gioi, ${ }^{32}$ J. Libby, ${ }^{15}$ Y. Liu, ${ }^{5}$ D. Liventsev, ${ }^{11}$ D. Matvienko, ${ }^{3}$ K. Miyabayashi, ${ }^{38}$ H. Miyata, ${ }^{44}$ R. Mizuk,${ }^{20,34}$ A. Moll, ${ }^{32,58}$ N. Muramatsu, ${ }^{51}$ R. Mussa, ${ }^{19}$ Y. Nagasaka, ${ }^{12}$ E. Nakano, ${ }^{46}$ M. Nakao, ${ }^{11}$ H. Nakazawa, ${ }^{39}$ M. Nayak,${ }^{15}$ E. Nedelkovska, ${ }^{32}$ C. Ng, ${ }^{62}$ M. Niiyama, ${ }^{26}$ N. K. Nisar, ${ }^{57}$ S. Nishida, ${ }^{11}$ O. Nitoh, ${ }^{65}$ S. Ogawa, ${ }^{59}$ S. Okuno, ${ }^{22}$ P. Pakhlov, ${ }^{20,34}$ G. Pakhlova, ${ }^{20}$ C. W. Park, ${ }^{55}$ H. Park, ${ }^{27}$ H. K. Park, ${ }^{27}$ T. K. Pedlar, ${ }^{30}$ T. Peng, ${ }^{52}$ R. Pestotnik, ${ }^{21}$ M. Petrič ${ }^{21}$ L. E. Piilonen, ${ }^{67}$ M. Ritter, ${ }^{32}$ M. Röhrken, ${ }^{23}$

A. Rostomyan, ${ }^{6}$ H. Sahoo, ${ }^{10}$ T. Saito, ${ }^{61}$ Y. Sakai, ${ }^{11}$ S. Sandilya, ${ }^{57}$ L. Santelj, ${ }^{21}$ T. Sanuki, ${ }^{61}$ V. Savinov, ${ }^{50}$ O. Schneider, ${ }^{28}$ G. Schnell, ${ }^{1,13}$ C. Schwanda ${ }^{17}$ D. Semmler, ${ }^{7}$ K. Senyo, ${ }^{69}$ O. Seon,${ }^{36}$ M. Shapkin, ${ }^{18}$ C. P. Shen, ${ }^{2}$ T.-A. Shibata, ${ }^{63}$ J.-G. Shiu, ${ }^{41}$ B. Shwartz, ${ }^{3}$ A. Sibidanov, ${ }^{56}$ Y.-S. Sohn, ${ }^{70}$ A. Sokolov, ${ }^{18}$ E. Solovieva, ${ }^{20}$ S. Stanič,${ }^{45}$ M. Starič,${ }^{21}$ M. Steder, ${ }^{6}$ M. Sumihama, ${ }^{8}$ T. Sumiyoshi, ${ }^{64}$ U. Tamponi, ${ }^{19,66}$ K. Tanida,${ }^{53}$ G. Tatishvili ${ }^{47}$ Y. Teramoto,${ }^{46}$ M. Uchida, ${ }^{63}$ S. Uehara, ${ }^{11}$ T. Uglov, ${ }^{20,35}$ Y. Unno, ${ }^{9}$ S. Uno, ${ }^{11}$ C. Van Hulse, ${ }^{1}$ P. Vanhoefer, ${ }^{32}$ G. Varner, ${ }^{10}$ A. Vinokurova,${ }^{3}$ V. Vorobyev, ${ }^{3}$

M. N. Wagner, ${ }^{7}$ C. H. Wang, ${ }^{40}$ M.-Z. Wang, ${ }^{41}$ P. Wang, ${ }^{16}$ M. Watanabe, ${ }^{44}$ Y. Watanabe, ${ }^{22}$ K. M. Williams, ${ }^{67}$ E. Won, ${ }^{25}$ Y. Yamashita, ${ }^{43}$ S. Yashchenko, ${ }^{6}$ Z. P. Zhang, ${ }^{52}$ V. Zhilich, ${ }^{3}$ V. Zhulanov, ${ }^{3}$ and A. Zupanc ${ }^{23}$

(Belle Collaboration)

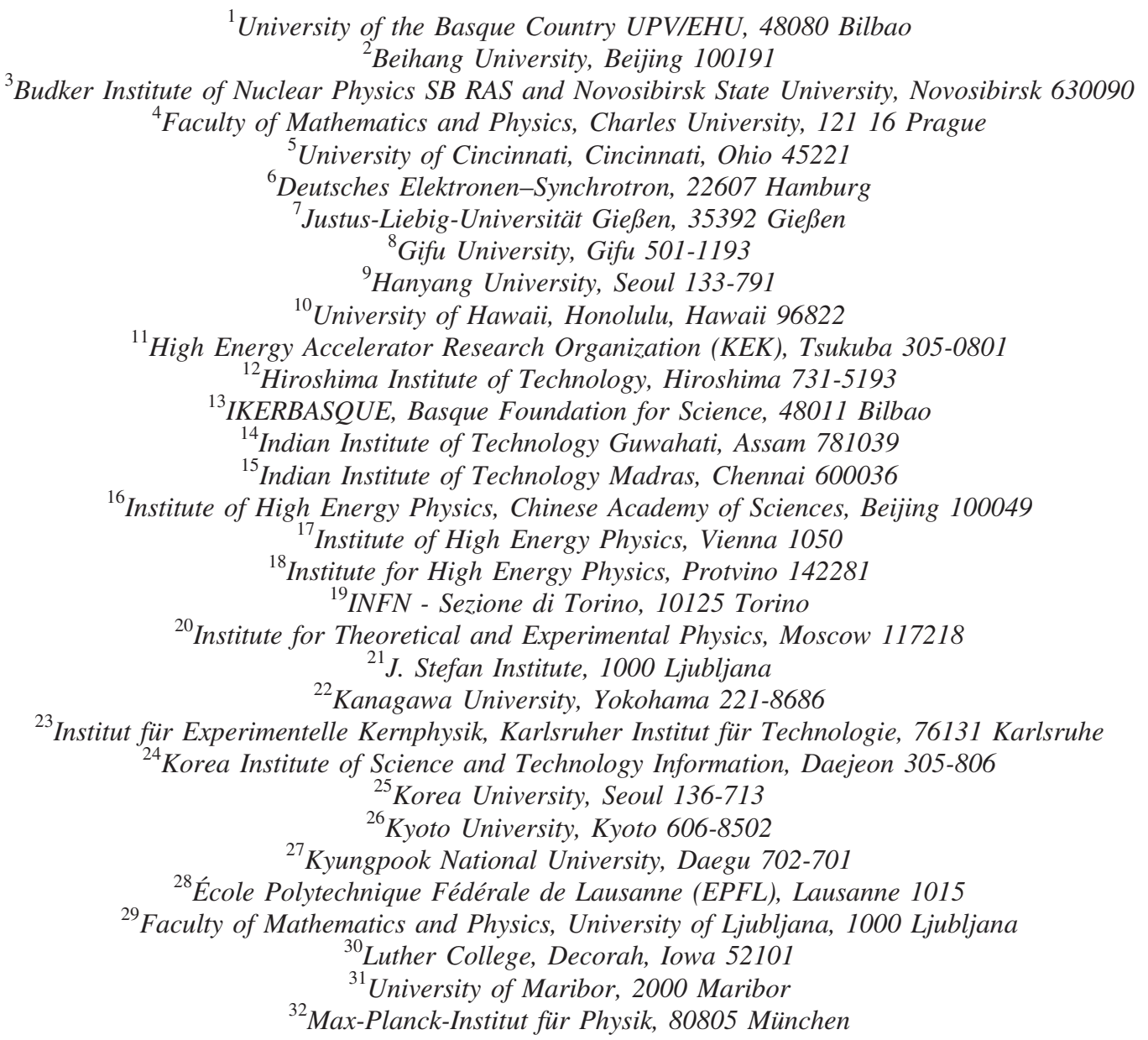




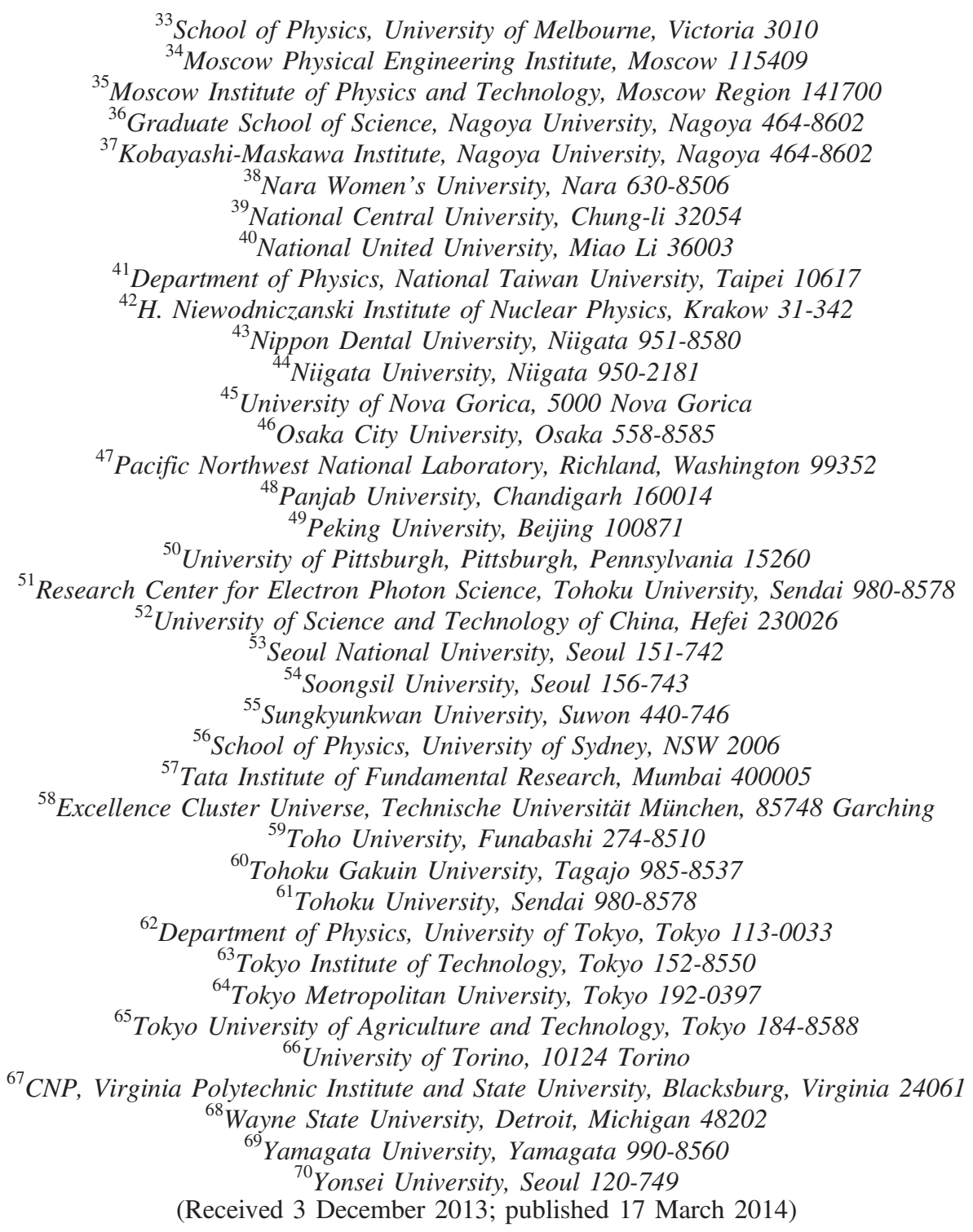

We report results of a study of doubly charmed baryons and charmed strange baryons. The analysis is performed using a $980 \mathrm{fb}^{-1}$ data sample collected with the Belle detector at the KEKB asymmetric-energy $e^{+} e^{-}$collider. We search for doubly charmed baryons $\Xi_{c c}^{+(+)}$with the $\Lambda_{c}^{+} K^{-} \pi^{+}\left(\pi^{+}\right)$and $\Xi_{c}^{0} \pi^{+}\left(\pi^{+}\right)$final states. No significant signal is observed. We also search for two excited charmed strange baryons, $\Xi_{c}(3055)^{+}$and $\Xi_{c}(3123)^{+}$with the $\Sigma_{c}^{++}(2455) K^{-}$and $\Sigma_{c}^{++}(2520) K^{-}$final states. The $\Xi_{c}(3055)^{+}$signal is observed with a significance of 6.6 standard deviations including systematic uncertainty, while no signature of the $\Xi_{c}(3123)^{+}$is seen. We also study properties of the $\Xi_{c}(2645)^{+}$and measure a width of $2.6 \pm 0.2$ (stat) \pm 0.4 (syst) $\mathrm{MeV} / c^{2}$, which is the first significant determination.

DOI: 10.1103/PhysRevD.89.052003

PACS numbers: 14.20.Lq, 14.20.-c, 14.20.Gk

\section{INTRODUCTION}

In recent years, there has been significant progress in charmed baryon spectroscopy, mainly by the Belle and $B A B A R$ experiments [1-8]. In particular, all the ground states of the single-charmed baryons predicted by the constituent quark model and several excited states have been observed [9].
However, there are no experimentally established doubly charmed baryons. The lightest doubly charmed baryon contains two charm quarks and one up or down quark $\left(\Xi_{c c}^{+}=c c d, \Xi_{c c}^{++}=c c u\right)$, and the spin-parity of the ground state is expected to be $\frac{1}{2}^{+}$. The mass of the $\Xi_{c c}$ has been extensively studied theoretically, and the prediction of the quark model ranges from $3.48 \mathrm{GeV} / c^{2}$ to $3.74 \mathrm{GeV} / c^{2}$ 
[10-22], whereas the mass predicted by lattice QCD ranges from $3.51 \mathrm{GeV} / c^{2}$ to $3.67 \mathrm{GeV} / c^{2}$ [23-27]. The cross sections of the $\Xi_{c c}$ production in the process $e^{+} e^{-} \rightarrow \Xi_{c c} X$ at $\sqrt{s}=10.58 \mathrm{GeV}$, where $X$ denotes the remaining particles produced in the fragmentation, is predicted to be $70 \mathrm{fb}$ in Ref. [28] and $230 \mathrm{fb}$ in Ref. [29]. The cross section of the pair production of the $c c$ and $\bar{c} \bar{c}$ diquarks is predicted to be $7 \mathrm{fb}$ [30].

There have been several experimental studies to search for the $\Xi_{c c}$. The SELEX collaboration reported evidence for the $\Xi_{c c}^{+}$in the $\Lambda_{c}^{+} K^{-} \pi^{+}$[31] and $p D^{+} K^{-}$ [32] final states with a mass of about $3.52 \mathrm{GeV} / c^{2}$ using a $600 \mathrm{GeV} / c$ charged hyperon beam. However, the results have not been supported by FOCUS [33], $B A B A R$ [34], Belle [2] nor LHCb [35]. The BABAR collaboration searched for the $\Xi_{c c}^{+(+)}$in the $\Lambda_{c}^{+} K^{-} \pi^{+}\left(\pi^{+}\right)$ and $\Xi_{c}^{0} \pi^{+}\left(\pi^{+}\right)$decay modes with a $232 \mathrm{fb}^{-1}$ data sample of $e^{+} e^{-}$collisions at or near the $\Upsilon(4 S)$. They found no evidence for the $\Xi_{c c}^{+(+)}$and set an upper limit on the product of the production cross section and branching fractions of $\Xi_{c c}$ and $\Lambda_{c}^{+}$or $\Xi_{c}^{0}$ to be a few fb, depending on the decay mode. In our search for the $\Xi_{c c}^{+}$in the $\Lambda_{c}^{+} K^{-} \pi^{+}$final state with a $462 \mathrm{fb}^{-1}$ data sample of Belle at or near the $\Upsilon(4 S)$ [2], Belle also found no evidence for the $\Xi_{c c}^{+}$and set an upper limit on $\sigma\left(e^{+} e^{-} \rightarrow \Xi_{c c}^{+} X\right) \times$ $\mathcal{B}\left(\Xi_{c c}^{+} \rightarrow \Lambda_{c}^{+} K^{-} \pi^{+}\right) / \sigma\left(e^{+} e^{-} \rightarrow \Lambda_{c}^{+} X\right)$ of $1.5 \times 10^{-4}$ with a $p^{*}\left(\Lambda_{c}^{+}\right)>2.5 \mathrm{GeV} / c$ requirement. Here, $p^{*}\left(\Lambda_{c}^{+}\right)$ is the momentum of the $\Lambda_{c}^{+}$in the center-of-mass (CM) frame.

In this paper, we report on an improved search for the $\Xi_{c c}$ in its weak decays to the $\Lambda_{c}^{+} K^{-} \pi^{+}\left(\pi^{+}\right)$and $\Xi_{c}^{0} \pi^{+}\left(\pi^{+}\right)$final states. The Belle collaboration has collected a data sample with a total integrated luminosity of $980 \mathrm{fb}^{-1}$, which is around two (four) times the statistics of the previous $\Xi_{c c}$ search by Belle [2] (BABAR [34]) and supersedes the results in Ref. [2]. Furthermore, in the previous studies, the $\Lambda_{c}^{+}$ and the $\Xi_{c}^{0}$ states have been reconstructed only from decay modes of $p K^{-} \pi^{+}$and $\Xi^{-} \pi^{+}$, respectively. We incorporate additional decay modes to improve the statistical sensitivity.

The same data sample can be used to study charmed strange baryons, as the $\Lambda_{c}^{+} K^{-} \pi^{+}$and the $\Xi_{c}^{0} \pi^{+}$final states are strong decay modes of excited $\Xi_{c}^{+}\left(\Xi_{c}^{*+}\right)$ states. The $B A B A R$ collaboration found two $\Xi_{c}^{*+}$ states, $\Xi_{c}(3055)^{+}$and $\Xi_{c}(3123)^{+}$, decaying to the $\Lambda_{c}^{+} K^{-} \pi^{+}$final state through intermediate $\Sigma_{c}(2455)^{++}$or $\Sigma_{c}(2520)^{++}$states using a data sample of $384 \mathrm{fb}^{-1}$ [6]. Their statistical significance was 6.4 standard deviations $(\sigma)$ and $3.6 \sigma$, respectively. A confirmation of these states in other experiments is necessary. The $\Xi_{c}^{0} \pi^{+}$is a strong decay mode of the $\Xi_{c}(2645)^{+}$. Currently, only the upper limit of $3.1 \mathrm{MeV} / c^{2}$ exists for its width [36]. In this paper, we also report on a search for the $\Xi_{c}(3055)^{+}$and $\Xi_{c}(3123)^{+}$in the $\Lambda_{c}^{+} K^{-} \pi^{+}$final state, and the measurement of the width of the $\Xi_{c}(2645)^{+}$.

The remaining sections of the paper are organized as follows. In Sec. II, the data samples and the Belle detector are described. In Sec. III, a study of the final states with $\Lambda_{c}^{+}$, i.e., the $\Xi_{c c}$ search and the $\Xi_{c}(3055)^{+}$and $\Xi_{c}(3123)^{+}$ search, are reported. In Sec. IV, a study of the final state with $\Xi_{c}^{0}$ i.e., the $\Xi_{c c}$ search and measurement of the width of the $\Xi_{c}(2645)^{+}$, are described. Finally, conclusions are given in Sec. V.

\section{DATA SAMPLES AND THE BELLE DETECTOR}

We use a data sample with a total integrated luminosity of $980 \mathrm{fb}^{-1}$ recorded with the Belle detector at the KEKB asymmetric-energy $e^{+} e^{-}$collider [37]. The data samples with different beam energies at or near the $\Upsilon(1 S)$ to $\Upsilon(5 S)$ are combined in this study. The beam energies and integrated luminosities are summarized in Table I. The luminosity-weighted average of $\sqrt{s}$ is $10.59 \mathrm{GeV}$.

The Belle detector is a large-solid-angle magnetic spectrometer that consists of a silicon vertex detector (SVD), a 50-layer central drift chamber (CDC), an array of aerogel threshold Cherenkov counters (ACC), a barrellike arrangement of time-of-flight scintillation counters (TOF), and an electromagnetic calorimeter comprised of $\mathrm{CsI}(\mathrm{Tl})$ crystals (ECL) located inside a superconducting solenoid coil that provides a $1.5 \mathrm{~T}$ magnetic field. An iron flux return located outside of the coil is instrumented to detect $K_{L}^{0}$ mesons and to identify muons (KLM). The detector is described in detail elsewhere [38]. Two inner detector configurations were used. A $2.0 \mathrm{~cm}$ radius beampipe and a 3-layer silicon vertex detector were used for the first sample of $156 \mathrm{fb}^{-1}$, while a $1.5 \mathrm{~cm}$ radius beampipe, a 4-layer silicon detector and a small-cell inner drift chamber were used to record the remaining $824 \mathrm{fb}^{-1}$ [39].

The selection of charged hadrons is based on information from the tracking system (SVD and CDC) and hadron identification system (CDC, ACC, and TOF). The charged proton, kaon, and pion that are not associated with longlived particles like $K_{S}^{0}, \Lambda$ and $\Xi^{-}$, are required to have a point of closest approach to the interaction point that is within $0.2 \mathrm{~cm}$ in the transverse $(r-\phi)$ direction and within $2 \mathrm{~cm}$ along the $z$-axis. (The $z$-axis is opposite the positron beam direction.) For each track, the likelihood values $\mathcal{L}_{p}$,

TABLE I. Summary of the integrated luminosities and beam energies.

\begin{tabular}{lccccc}
\hline \hline$\sqrt{s}$ & $\Upsilon(5 S) /$ near it & $\Upsilon(4 S) /$ near it & $\Upsilon(3 S) /$ near it & $\Upsilon(2 S) /$ near it & $\Upsilon(1 S) /$ near it \\
\hline Integrated luminosity $\left(\mathrm{fb}^{-1}\right)$ & $121.0 / 29.3$ & $702.6 / 89.5$ & $2.9 / 0.3$ & $24.9 / 1.8$ & $5.7 / 1.8$ \\
\hline \hline
\end{tabular}


$\mathcal{L}_{K}$, and $\mathcal{L}_{\pi}$ are provided for the assumption of proton, kaon, and pion, respectively, from the hadron identification system, based on the ionization energy loss in the CDC, the number of detected Cherenkov photons in the ACC, and the time of flight measured by the TOF. The likelihood ratio is defined as $\mathcal{L}(i: j)=\mathcal{L}_{i} /\left(\mathcal{L}_{i}+\mathcal{L}_{j}\right)$ and a track is identified as a proton if the likelihood ratios $\mathcal{L}(p: \pi)$ and $\mathcal{L}(p: K)$ are greater than 0.6. A track is identified as a kaon if the likelihood ratios $\mathcal{L}(K: \pi)$ and $\mathcal{L}(K: p)$ are greater than 0.6. A track is identified as a pion if the likelihood ratios $\mathcal{L}(\pi: K)$ and $\mathcal{L}(\pi: p)$ are greater than 0.6. In addition, electron $\left(\mathcal{L}_{e}\right)$ likelihood is provided based on information from the ECL, ACC, and CDC [40]. A track with an electron likelihood greater than 0.95 is rejected.

The momentum averaged efficiencies of hadron identification are about $90 \%, 90 \%$, and $93 \%$ for pions, kaons, and protons, respectively. The momentum averaged probability to misidentify a pion (kaon) track as a kaon (pion) track is about $9(10) \%$, and the momentum averaged probability to misidentify a pion or kaon track as a proton track is about $5 \%$.

We use a Monte-Carlo (MC) simulation events generated with EVTGEN [41], JETSET [42] with final QED final state radiation by PHOTOS [43] and then processed by a GEANT3 [44] based detector simulation to obtain the reconstruction efficiency and the mass resolution.

\section{FINAL STATE WITH THE $\Lambda_{C}^{+}$}

In this section, the analysis using the final states with the $\Lambda_{c}^{+}$baryon is described. Reconstruction of the $\Lambda_{c}^{+}$candidate is explained first, followed by the description of the $\Xi_{c c}^{+(+)}$search in its decay into $\Lambda_{c}^{+} K^{-} \pi^{+}\left(\pi^{+}\right)$and the study of two charmed strange baryons, $\Xi_{c}(3055)^{+}$and $\Xi_{c}(3123)^{+}$. Throughout this paper, the selection criteria are determined to maximize the figure of merit (FOM), defined as $\varepsilon / \sqrt{N_{\mathrm{bg}}}$, where $\varepsilon$ is the $\Xi_{c c}$ efficiency for the selection criteria and $N_{\text {bg }}$ is the number of background events under the signal peak except for the scaled momentum selection for the $\Xi_{c}(3055)^{+}$and $\Xi_{c}(3123)^{+}$search, which followed $B A B A R$ 's analysis. The distribution of background events is estimated based on data. When the selection criteria are determined, we hide the possible signal peak by smearing the invariant mass of the $\Xi_{c c}$ candidates event by event with a Gaussian having a $50 \mathrm{MeV} / \mathrm{c}^{2}$ width in order to avoid any biases.

\section{A. Reconstruction of the $\Lambda_{c}^{+}$}

The $\Lambda_{c}^{+}$candidates are reconstructed in the $p K^{-} \pi^{+}$and $p K_{S}^{0}$ decay modes [45]. The $K_{S}^{0}$ candidate is reconstructed from its decay into $\pi^{+} \pi^{-}$. A pair of oppositely charged pions that have an invariant mass within $8 \mathrm{MeV} / c^{2}$ of the nominal $K_{S}^{0}$ mass, which corresponds to approximately $3.5 \sigma$ of the mass resolution, is used. Two pion tracks are fitted to a common vertex. The result of the fit is used to suppress misreconstructed $K_{S}^{0}$ candidates and to perform further vertex fit of the $\Lambda_{c}^{+} \rightarrow p K_{S}^{0}$. The vertex of the two pions for the $K_{S}^{0}$ is required to be displaced from the interaction point (IP) in the direction of the pion pair momentum [46]. The daughters of the $\Lambda_{c}^{+}$are fitted to a common vertex; the invariant mass of the daughters must be within $5(6) \mathrm{MeV} / c^{2}$, or $1.5 \sigma$, nominal $\Lambda_{c}^{+}$mass for the $p K^{-} \pi^{+}\left(p K_{S}^{0}\right)$ decay mode. The $\chi^{2}$ value of the common vertex fit of the $\Lambda_{c}^{+}$is required to be less than 50 . For the remaining candidate, a mass constraint fit to the $\Lambda_{c}^{+}$mass is performed to improve the momentum resolution. As the signal-to-background ratio for the $\Lambda_{c}^{+}$candidates is similar for the $p K^{-} \pi^{+}$and $p K_{S}^{0}$ decay modes, they are combined in the following analysis. By including the $p K_{S}^{0}$ mode in addition to the $p K^{-} \pi^{+}$mode, the yield of the $\Lambda_{c}^{+}$is increased by about $20 \%$.

\section{B. Search for doubly charmed baryons in $\Lambda_{c}^{+} \boldsymbol{K}^{-} \boldsymbol{\pi}^{+}\left(\boldsymbol{\pi}^{+}\right)$}

We search for the $\Xi_{c c}^{+(+)}$in its decay into $\Lambda_{c}^{+} K^{-} \pi^{+}\left(\pi^{+}\right)$in the mass range of 3.2-4.0 GeV/c $c^{2}$. The expected mass resolution of the $\Xi_{c c}$ estimated from MC is $2.0-3.5 \mathrm{MeV} / c^{2}$, depending on the mass of the $\Xi_{c c}$ (degrading with increasing mass). In order to reduce the combinatorial background, a selection on the scaled momentum $x_{p}=$ $p^{*} \sqrt{s / 4-m^{2}}$ is used, where $p^{*}$ is the CM momentum of a $\Xi_{c c}$ candidate and $s$ is CM energy squared and $m$ is mass of the $\Xi_{c c}$ candidate. As there is no measurement of the $x_{p}$ spectrum for $\Xi_{c c}$ production, we assume it to be the same as that of the $\Lambda_{c}^{+}$, which has been precisely measured [47]. The $x_{p}$ spectrum is represented by a smooth polynomial function and is used to generate a MC sample for the $\Xi_{c c}$ signal. Decays of the $\Xi_{c c}$ and $\Lambda_{c}^{+}$are generated according to the available phase space distribution. The number of background events as a function of the $x_{p}$ cut is estimated based on smeared data. The FOM as a function of the $x_{p}$ cut is surveyed in the search region. The optimization procedure yields $0.5<x_{p}<1.0$ regardless of the $\Xi_{c c}$ mass. To check the validity of our analysis, we independently examine the $x_{p}$ spectrum of the $\Lambda_{c}^{+}$and confirm that it is consistent with that presented in Ref. [47].

Figure 1(a) and 1(b) show the $M\left(\Lambda_{c}^{+} K^{-} \pi^{+}\right)$and $M\left(\Lambda_{c}^{+} K^{-} \pi^{+} \pi^{+}\right)$distributions, respectively, for data after all the event selections are applied. No significant signal is seen in the data for either $\Xi_{c c}^{+}$or $\Xi_{c c}^{++}$. The statistical significance for a given mass is evaluated with an unbinned extended maximum likelihood (UML) fit. The probability density function (PDF) for the signal is described with signal MC generated for each given $\Xi_{c c}$ mass, whereas a third-order polynomial function is used as the background PDF. The statistical significance is defined as $\sqrt{-2 \ln \left(\mathcal{L}_{0} / \mathcal{L}\right)}$, where $\mathcal{L}_{0}$ is the likelihood for the fit without the signal component and $\mathcal{L}$ is the likelihood for the fit with the signal component included. The significance is evaluated for the $\Xi_{c c}$ mass 

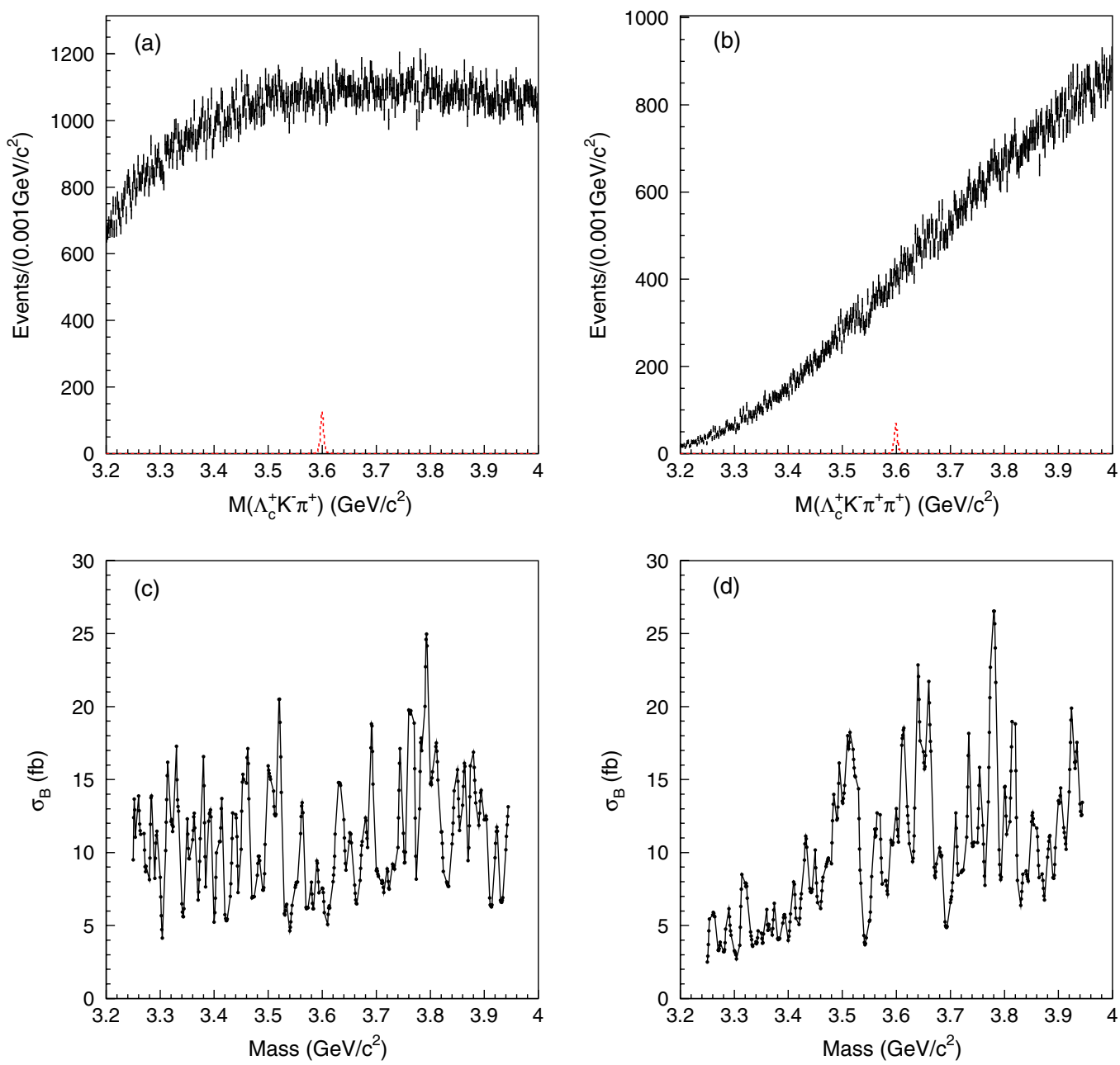

FIG. 1 (color online). Invariant mass distribution of the $\Xi_{c c}$ candidates for (a) $M\left(\Lambda_{c}^{+} K^{-} \pi^{+}\right.$), (b) $M\left(\Lambda_{c}^{+} K^{-} \pi^{+} \pi^{+}\right.$); the vertical error bars are from data and the dashed histogram are from signal $\mathrm{MC}$ for the $\Xi_{c c}$ signal generated with a mass of $3.60 \mathrm{GeV} / c^{2}$ and a production cross section $\sigma\left(e^{+} e^{-} \rightarrow \Xi_{c c}^{+(+)} X\right)$ of $500 \mathrm{fb}$ and $\mathcal{B}\left(\Xi_{c c}^{+(+)} \rightarrow \Lambda_{c}^{+} K^{-} \pi^{+}\left(\pi^{+}\right)\right)$of $5 \% .95 \%$ C.L. upper limit of $\sigma_{\mathcal{B}}$ as a function of the mass with a $1 \mathrm{MeV} / c^{2}$ step for (c) $\Xi_{c c}^{+}$and (d) $\Xi_{c c}^{++}$.

scanned with a $1 \mathrm{MeV} / \mathrm{c}^{2}$ step in the search region. None of the mass points give a significance exceeding the $3 \sigma$ level.

The 95\% confidence level (C.L.) upper limit for the product of the cross section and branching fraction to the $\Lambda_{c} K^{-} \pi^{+}\left(\pi^{+}\right)$state produced with the $0.5<x_{p}<1.0$ condition,

$$
\sigma_{\mathcal{B}} \equiv \sigma\left(e^{+} e^{-} \rightarrow \Xi_{c c}^{+(+)} X\right)
$$

is evaluated. Here, $L$ is the total integrated luminosity, $N_{\text {sig }}$ is the $\Xi_{c c}$ signal yield, $\mathcal{B}_{p K^{-} \pi^{+}}$is the branching fraction of the $\Lambda_{c}^{+} \rightarrow p K^{-} \pi^{+}$(which amounts to $0.050 \pm 0.013$ ), $\mathcal{B}_{p K_{S}}$ is the branching fraction of $\Lambda_{c}^{+} \rightarrow p K_{S}^{0}$ measured relative to the $p K^{-} \pi^{+}$mode $\left(\mathcal{B}_{p K_{S}} / \mathcal{B}_{p K^{-} \pi^{+}}=0.24 \pm 0.02\right)$, and $\varepsilon_{p K^{-} \pi^{+}\left(p K_{S}\right)}$ is the reconstruction efficiency for the $\Lambda_{c}^{+} \rightarrow$ $p K^{-} \pi^{+}\left(\Lambda_{c}^{+} \rightarrow p K_{S}^{0}\right)$ decay mode evaluated as a function of the $\Xi_{c c}$ mass. The efficiencies for the $\Xi_{c c}^{+(+)}$as a function of their masses are shown in Fig. 2. The factor of two in the denominator comes from inclusion of the charge-conjugate mode. By including this factor, our measurement can be compared with the theoretical predictions [28,29]; while to compare with the prediction in Ref. [30], it is necessary to multiply our $\sigma_{\mathcal{B}}$ measurement by 2 because they predicted the cross section of the pair production of the $c c$ and $\bar{c} \bar{c}$ diquarks. In BABAR's measurement [34], they do not introduce the factor of two, i.e., they report an upper limit for the sum of the $\sigma\left(e^{+} e^{-} \rightarrow \Xi_{c c}^{+(+)} X\right)$ and its chargeconjugate mode). Therefore, our measurement should be doubled when comparing with $B A B A R$ 's result. We note that the cross section reported here and elsewhere in this paper is a visible cross section (i.e., a radiative correction is not applied.

The upper limit is evaluated following the Bayesian approach. First, we scan the likelihood profile by determining the likelihood values as a function of the $\sigma_{\mathcal{B}}\left(\mathcal{L}\left(\sigma_{\mathcal{B}}\right)\right)$, 

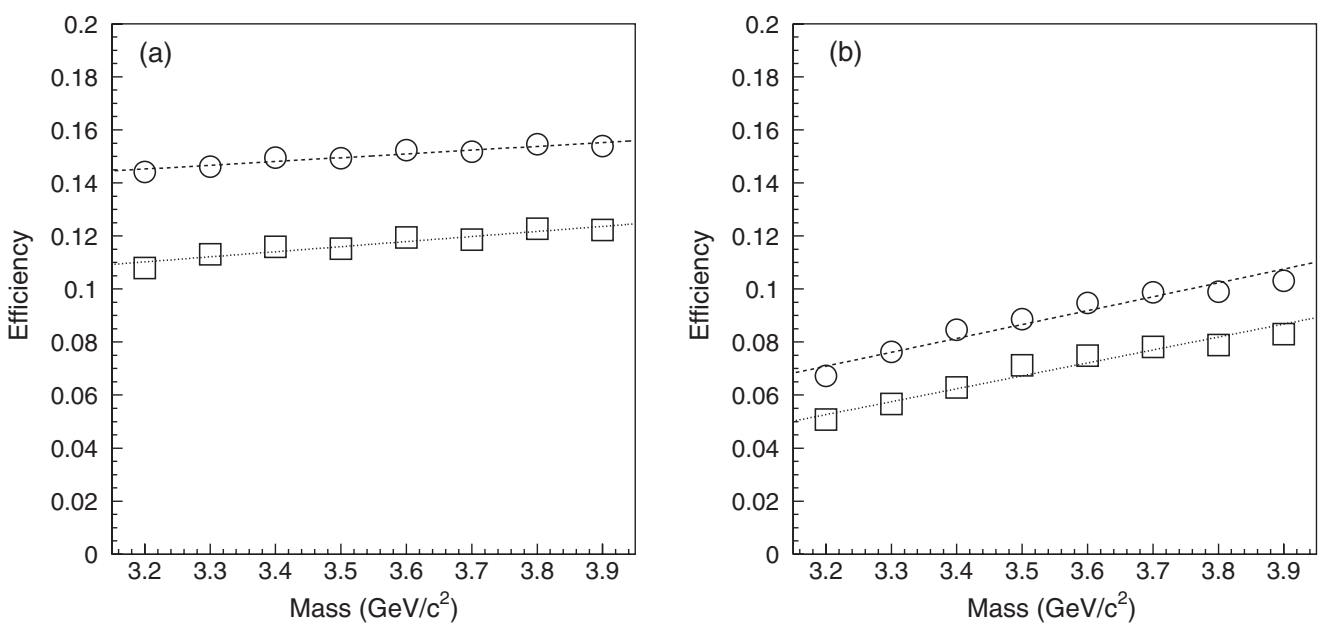

FIG. 2. Reconstruction efficiency as a function of the $\Xi_{c c}$ mass, for (a) $\Xi_{c c}^{+}$, (b) $\Xi_{c c}^{++}$. Circular points are for $\Lambda_{c}^{+} \rightarrow p K^{-} \pi^{+}$and square points are for $\Lambda_{c}^{+} \rightarrow p K_{S}^{0}$. The lines show the result of the fit with a linear function.

varying $N_{\text {sig }}$ from zero up to the $N_{\text {sig }}$ value for which the likelihood drops to zero. Then, $\mathcal{L}\left(\sigma_{\mathcal{B}}\right)$ is convolved with a Gaussian whose width equals the systematic uncertainties of $\sigma_{\mathcal{B}}$. The $\sigma_{\mathcal{B}}$ value for which the integral (starting from $\sigma_{\mathcal{B}}=0$ ) becomes $95 \%$ of the entire area is regarded as the 95\% C.L. upper limit.

We consider the following systematic uncertainties in the $\Xi_{c c}$ search. The systematic uncertainty due to the efficiency of pion and kaon identification is estimated from the ratio of the yield of the $D^{*+} \rightarrow D^{0} \pi^{+}, D^{0} \rightarrow K^{-} \pi^{+}$with and without the pion/kaon identification requirements for data and $\mathrm{MC}$. The difference of the ratio between data and $\mathrm{MC}$ is corrected and the statistical error of the ratio is regarded as the systematic uncertainty. The systematic uncertainty due to the efficiency of proton identification is estimated using the ratio of the yield of the $\Lambda \rightarrow p \pi^{-}$with and without the proton identification requirement. The difference of the ratio between data and $\mathrm{MC}$ is corrected and the statistical error of the ratio is regarded as the systematic uncertainty. The systematic uncertainty due to the charged track reconstruction efficiency is estimated using the decay chain $D^{*+} \rightarrow \pi^{+} D^{0}, D^{0} \rightarrow \pi^{+} \pi^{-} K_{S}^{0}$, and $K_{S}^{0} \rightarrow \pi^{+} \pi^{-}$, where $K_{S}^{0} \rightarrow \pi^{+} \pi^{-}$is either partially or fully reconstructed. The ratio of the yields for partially and fully reconstructed signals in data and $\mathrm{MC}$ is compared, and the difference is taken as the systematic uncertainty. This amounts to $0.35 \%$ per track. The systematic uncertainty of the total integrated luminosity is $1.4 \%$. To check the systematic error due to the signal PDF, we compare the mass resolution of the $\Lambda_{c}^{+}$in data and $\mathrm{MC}$. We find that the resolution for data is $5 \%$ larger than in MC. To monitor the effect of this discrepancy, we perform a pseudoexperiment test in which we extract the signal yield with correct PDF and one that is narrower by $5 \%$. The largest difference of $3 \%$ measured in this test is regarded as the systematic uncertainty. The systematic uncertainty related to the $\Lambda_{c}^{+}$branching fraction is propagated from the errors taken from the PDG [9]. To estimate the systematic uncertainty of the reconstruction efficiency due to the possible difference of $x_{p}$ spectrum between our assumption (the same as that of $\Lambda_{c}^{+}$) and the actual one, we examine the $x_{p}$ dependence of the reconstruction efficiency. The root mean square of the reconstruction efficiency in the region of $0.5<x_{p}<1.0$ is regarded as the systematic uncertainty. The elements of the systematic uncertainty for the measurement of the $\sigma_{\mathcal{B}}$ are enumerated in the first and second columns of Table II.

Figures 1(c) and 1(d) show the 95\% C.L. upper limit on $\sigma_{\mathcal{B}}$ for $\Xi_{c c}^{+}$and $\Xi_{c c}^{++}$, respectively, as a function of the mass

TABLE II. Summary of the systematic uncertainties in the $\sigma_{\mathcal{B}}$ and $\sigma_{\mathcal{B}^{2}}$ measurement (\%).

\begin{tabular}{lccccc}
\hline \hline Source & $\Xi_{c c}^{+}$with $\Lambda_{c}^{+}$ & $\Xi_{c c}^{++}$with $\Lambda_{c}^{+}$ & $\Xi_{c}(3123)^{+}$ & $\Xi_{c c}^{+}$with $\Xi_{c}^{0}$ & $\Xi_{c c}^{++}$with $\Xi_{c}^{0}$ \\
\hline Particle ID & 2.0 & 2.4 & 2.0 & 3.5 & 3.8 \\
Tracking & 1.8 & 2.1 & 1.8 & 1.8 & 2.1 \\
Signal PDF & 3.5 & 3.5 & 28.0 & 3.5 & 3.5 \\
Luminosity & 1.4 & 1.4 & 1.4 & 1.4 & 0.7 \\
$\mathcal{B}$ & 26.0 & 26.0 & 1.6 & 6.0 & 5.7 \\
$x_{p}$ & 2.1 & 2.3 & 1.2 & 4.3 & 4.3 \\
$N_{\Xi_{c}^{i}(2645)^{+}}$ & $\ldots$ & $\ldots$ & 26.2 & 9.2 & 9.2 \\
total & 26.5 & 26.6 & & & \\
\hline \hline
\end{tabular}


with a $1 \mathrm{MeV} / c^{2}$ step. The upper limit is in the range of 4.1-25.0 fb for the $\Xi_{c c}^{+}$and 2.5-26.5 fb for the $\Xi_{c c}^{++}$.

\section{Search for the $\Xi_{c}^{+}(\mathbf{3 0 5 5})$ and $\Xi_{c}^{+}(\mathbf{3 1 2 3})$}

In this section, a search for the $\Xi_{c}^{+}(3055)$ and $\Xi_{c}^{+}(3123)$ is described. Here, we require $x_{p}$ to be greater than 0.7. In the analysis by $B A B A R$ [6], they required $p^{*}\left(\Lambda_{c}^{+} K^{-} \pi^{+}\right)>$ $2.9 \mathrm{GeV} / c$, which is similar to our $x_{p}$ cut as illustrated by the $p^{*}\left(\Lambda_{c}^{+} K^{-} \pi^{+}\right)$distribution, with the $x_{p}$ cut and $2.9 \mathrm{GeV} / c^{2}<M\left(\Lambda_{c}^{+} K^{-} \pi^{+}\right)<3.2 \mathrm{GeV} / c^{2}$ required as shown in Fig. 3(a). Figure 3(b) shows the $M\left(\Lambda_{c}^{+} \pi^{+}\right)$ distribution, where contributions from the $\Sigma_{c}(2455)^{++}$ and the $\Sigma_{c}(2520)^{++}$baryons are clearly visible. We select the $\Sigma_{c}(2455)^{++}\left[\Sigma_{c}(2520)^{++}\right]$region by requiring
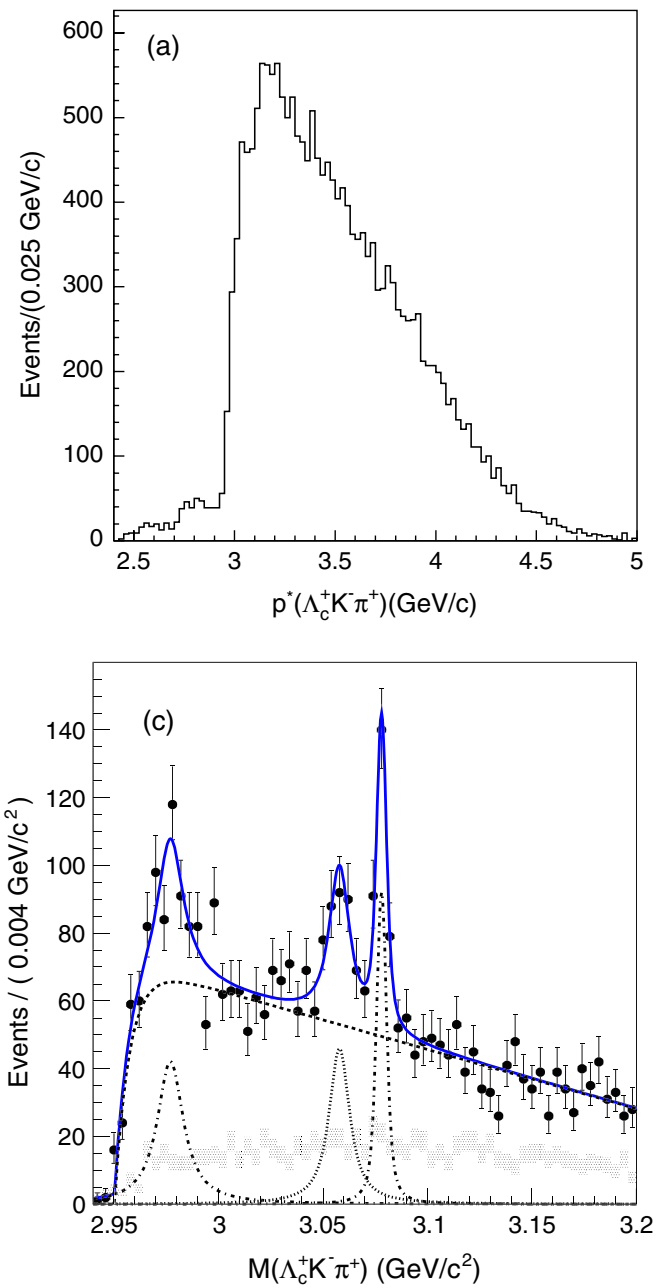

$\left|M\left(\Lambda_{c}^{+} \pi^{+}\right)-m_{\Sigma_{f}^{++}}\right|<5(18) \mathrm{MeV} / c^{2}$, where $m_{\Sigma_{c}^{++}}$is the nominal mass of the $\Sigma_{c}(2455)^{++}$or $\Sigma_{c}(2520)^{++}$.

Figure 3(c) shows the $M\left(\Lambda_{c}^{+} K^{-} \pi^{+}\right)$distribution for the $\Sigma_{c}(2455)^{++}$signal region together with the same plot for the $\Sigma_{c}(2455)^{++}$sideband region, defined as $\left|M\left(\Lambda_{c}^{+} \pi^{+}\right)-\left(m_{\Sigma_{c}(2455)^{++}} \pm 15 \mathrm{MeV} / c^{2}\right)\right|<5 \mathrm{MeV} / c^{2}$. Clear peaks corresponding to the $\Xi_{c}(2980)^{+}, \Xi_{c}(3055)^{+}$, and $\Xi_{c}(3080)^{+}$are seen. To obtain the statistical significance of the $\Xi_{c}(3055)^{+}$, an UML fit is applied. PDFs for the $\Xi_{c}^{*+}$ components are represented by a Breit-Wigner line shape convolved with a Gaussian to account for the invariant-mass resolution $\left(\sigma_{\mathrm{res}}\right)$. Using the signal MC events, we estimate $\sigma_{\text {res }}$ to vary from 1.2 to $1.8 \mathrm{MeV} / c^{2}$, depending on the masses of the $\Xi_{c}^{*+}$ states. The width and mean of the Breit-Wigner functions are treated as free
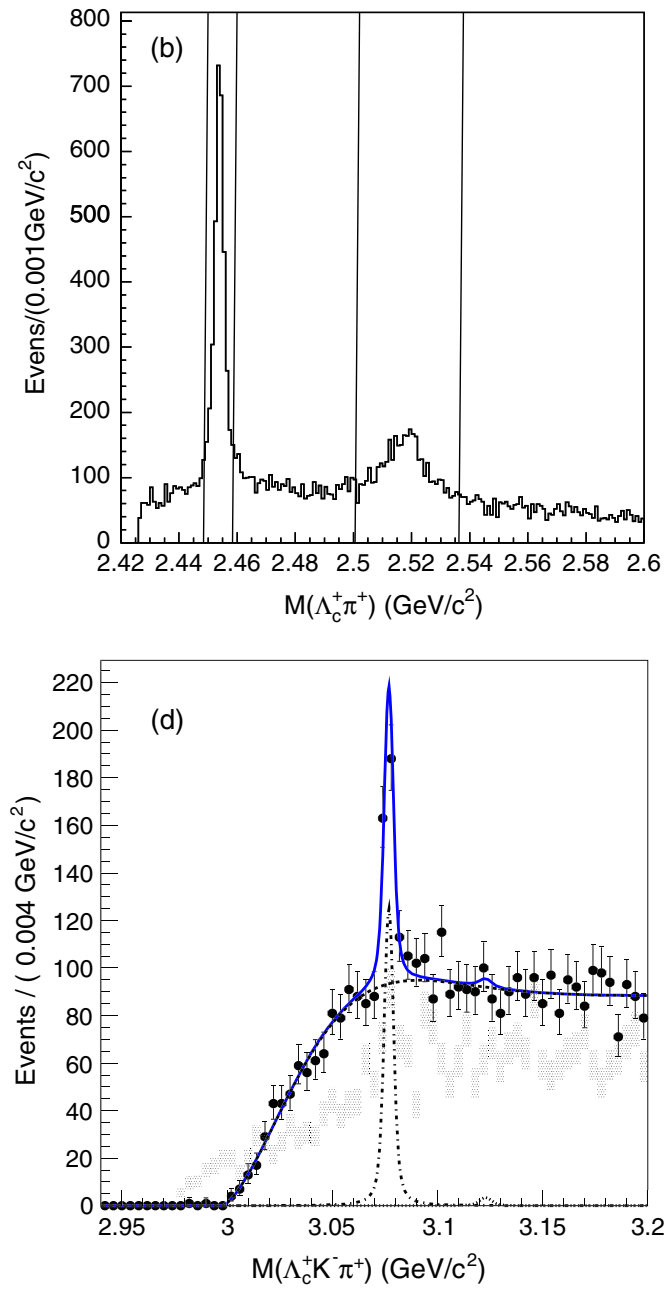

FIG. 3 (color online). (a) The $p^{*}\left(\Lambda_{c}^{+} K^{-} \pi^{+}\right)$distribution from data. (b) The $M\left(\Lambda_{c}^{+} \pi^{+}\right)$distribution. The vertical lines show the selected regions of the $\Sigma_{c}(2455)^{++}$and $\Sigma_{c}(2520)^{++}$. (c) The $M\left(\Lambda_{c}^{+} K^{-} \pi^{+}\right)$distribution with $\Sigma_{c}(2455)^{++}$selection. The dots with error bars show the distribution for the $\Sigma_{c}(2455)^{++}$selected region whereas the rectangles show the distribution for the $\Sigma_{c}(2455)^{++}$sideband region. The solid line shows the fit result. The dashed, dotted, and dash-dotted lines show the contributions from the background, $\Xi_{c}(3055)^{+}$, and $\Xi_{c}(2980)^{+}$, or $\Xi_{c}(3080)^{+}$, respectively. (d) The $M\left(\Lambda_{c}^{+} K^{-} \pi^{+}\right)$distribution with $\Sigma_{c}(2520)^{++}$selection. The dots with error bars show the distribution for $\Sigma_{c}(2520)^{++}$selected region whereas the rectangles show the distribution for the $\Sigma_{c}(2520)^{++}$ sideband region. The solid line shows the fit result. The dashed, dotted, and dash-dotted lines show the contributions from the background, $\Xi_{c}(3123)^{+}$, and $\Xi_{c}(3080)^{+}$, respectively. 
parameters. The background PDF, $f_{1}(x)$, is modeled with a threshold function:

$$
\begin{aligned}
f_{1}(x)= & 1-\exp \left(\left(x-x_{0}\right) / \delta_{m}\right)\left(x / x_{0}\right)^{a} \\
& +b\left(x / x_{0}-1\right) \quad\left(\text { if } x>x_{0}\right) \\
f_{1}(x)= & 0 \quad\left(\text { if } x<x_{0}\right),
\end{aligned}
$$

where $a, b, x_{0}$, and $\delta_{m}$ are free parameters in the fit.

The fit result is shown in Fig. 3(c). To estimate the statistical significance of the $\Xi_{c}(3055)^{+}$, we compare the likelihood values for the fits with and without the $\Xi_{c}(3055)^{+}$component. The obtained $-2 \ln \left(\mathcal{L}_{0} / \mathcal{L}\right)$ value is 54.7. By taking into account the change of the number of degrees of freedom ( $n d f)$ by the inclusion of the $\Xi_{c}(3055)^{+}$component, the statistical significance of the $\Xi_{c}(3055)^{+}$becomes $6.8 \sigma$. The $\chi^{2} / n d f$ of the fit with the $\Xi_{c}(3055)^{+}$component, for the binning of Fig. 3(c), is $54.8 / 61$.

Figure 3(d) shows the $M\left(\Lambda_{c}^{+} K^{-} \pi^{+}\right)$distribution for the $\Sigma_{c}(2520)^{++}$selected region together with the same plot for the $\Sigma_{c}(2520)^{++}$sideband region, defined as $\left|M\left(\Lambda_{c}^{+} \pi^{+}\right)-\left(m_{\Sigma_{c}(2520)^{++}} \pm 27 \mathrm{MeV} / c^{2}\right)\right|<12 \mathrm{MeV} / c^{2}$. A clear peak corresponding to the $\Xi_{c}(3080)^{+}$is seen, while no peak structure is seen in the mass near $3.123 \mathrm{GeV} / c^{2}$. An UML fit is applied to extract the signal yield. Again, the $\Xi_{c}^{*+}$ components are represented by a Breit-Wigner function convolved with a Gaussian. For the $\Xi_{c}(3080)^{+}$ component, the mass and width of the Breit-Wigner are treated as free parameters; while for the $\Xi_{c}(3123)^{+}$ component, the mass and width are fixed to the values obtained in Ref.[6]. The background shape, $f_{2}(x)$, is assumed to be:

$$
\begin{aligned}
f_{2}(x)= & \left(1-\exp \left(\left(x-x_{0}\right)^{2} / \delta_{m}\right)\right)\left(x / x_{0}\right)^{a} \\
& +b\left(x / x_{0}-1\right)+c\left(\left(x / x_{0}\right)^{2}-1\right) \quad\left(\text { if } x>x_{0}\right) \\
f_{2}(x)= & 0 \quad\left(\text { if } x<x_{0}\right),
\end{aligned}
$$

where $a, b, c, x_{0}$, and $\delta_{m}$ are fit parameters. The $\chi^{2} / n d f$ of the fit with the $\Xi_{c}(3123)^{+}$component for the binning of Fig. 3(d) is 28.6/42. The yield of the $\Xi_{c}(3123)^{+}$is $8 \pm 22$ events, which is consistent with zero. Hence, a 95\% C.L. upper limit for the production cross section is evaluated with the method described in the previous section. To directly compare with the $B A B A R$ result in Ref. [6], the upper limit for the product of the cross section and branching fraction of $\Lambda_{c}^{+}$produced with $x_{p}>0.7$ condition,

$$
\sigma_{\mathcal{B} \Lambda_{c}^{+}} \equiv \sigma\left(e^{+} e^{-} \rightarrow \Xi_{c}(3123)^{+} X\right) \times \mathcal{B}\left(\Lambda_{c}^{+} \rightarrow p K^{-} \pi^{+}\right)
$$

is evaluated. As in Ref. [6], we assume $\mathcal{B}\left(\Xi_{c}(3123)^{+} \rightarrow\right.$ $\left.\Sigma_{c}(2520)^{++} K^{-}\right)$is equal to 1 .
To take the uncertainty of the $\Xi_{c}(3123)^{+}$mass and width from Ref. [6] into account, we perform a pseudoexperiment test. The background and $\Xi_{c}(3080)^{+}$contributions are generated with statistics similar to data and based on the fit result. The $\Xi_{c}(3123)^{+}$component is generated with mass and width changed by $\pm 1 \sigma$, corresponding to their measured uncertainties $(3122.9 \pm 1.3 \pm$ $0.4 \mathrm{MeV} / c^{2}$ for the mass and $4.4 \pm 3.4 \pm 1.7 \mathrm{MeV} / c^{2}$ for the width). The yield of $\Xi_{c}(3123)^{+}$is extracted by fitting pseudoexperiment data with the procedure used for data. The ratio of the generated and extracted yield is regarded as a systematic uncertainty. Because the error of the width is relatively large, its systematic uncertainty contribution is dominant $(28 \%)$. All of the systematic errors are summarized in the third column of Table II. The $95 \%$ C.L. upper limit on $\sigma_{\mathcal{B} \Lambda_{c}^{+}}$is $0.17 \mathrm{fb}$. As in the case of the $\Xi_{c c}$ cross section, the measurement in Ref. [6] does not introduce a factor of two for the $\sigma_{\mathcal{B} \Lambda_{c}^{+}}$calculation. Therefore, we should double our measurement, which results in $0.34 \mathrm{fb}$, when comparing with $B A B A R$ 's result. The value is much smaller than that quoted in Ref. [6] $(1.6 \pm 0.6 \pm 0.2 \mathrm{fb})$.

The systematic uncertainties of the masses and widths of the $\Xi_{c}^{*+}$ and stability of the statistical significance of the $\Xi_{c}(3055)^{+}$are studied by the following fitting configurations. The systematic uncertainties due to the signal PDF are studied by varying $\sigma_{\text {res }}$ by $5 \%$. The systematic uncertainties due to possible interference between the $\Xi_{c}(3055)^{+}$and $\Xi_{c}(3080)^{+}$are studied by fitting the distribution with an additional phase parameter between the two Breit-Wigner amplitudes. The systematic uncertainty due to the background shape is studied by fitting the mass spectra with a second-order polynomial as a background PDF in the range of $3.005-3.200 \mathrm{GeV} / c^{2}$. In none of these fitting configurations does the statistical significance of the $\Xi_{c}(3055)^{+}$ fall below $6.6 \sigma$. We apply cut conditions of $x_{p}>0.6$ and $x_{p}>0.8$ instead of $x_{p}>0.7$ and reextract the masses and widths of the $\Xi_{c}^{*+}$ states. The differences from the default cut condition are regarded as systematic uncertainties. The measured masses, widths, and yields of the three $\Xi_{c}^{*+}$ states are summarized in Table III. All of these measurements are consistent with previous Belle measurement [2] within $2.5 \sigma$ and with the $B A B A R$ measurement [6] within $2.0 \sigma$.

TABLE III. The measured masses and widths of the three $\Xi_{c}^{*+}$ states. The first error is statistical and second is systematic.

\begin{tabular}{lcrc}
\hline \hline Particle & Mass $\left(\mathrm{MeV} / c^{2}\right)$ & \multicolumn{1}{c}{ Width $\left(\mathrm{MeV} / c^{2}\right)$} & Yield \\
\hline$\Xi_{c}(2980)^{+}$ & $2974.9 \pm 1.5 \pm 2.1$ & $14.8 \pm 2.5 \pm 4.1$ & $244 \pm 39$ \\
$\Xi_{c}(3055)^{+}$ & $3058.1 \pm 1.0 \pm 2.1$ & $9.7 \pm 3.4 \pm 3.3$ & $199 \pm 46$ \\
$\Xi_{c}(3080)^{+}\left(\Sigma_{c}\right)$ & $3077.9 \pm 0.4 \pm 0.7$ & $3.2 \pm 1.3 \pm 1.3$ & $185 \pm 31$ \\
$\Xi_{c}(3080)^{+}\left(\Sigma_{c}^{*}\right)$ & $3076.9 \pm 0.3 \pm 0.2$ & $2.4 \pm 0.9 \pm 1.6$ & $210 \pm 30$ \\
\hline \hline
\end{tabular}




\section{FINAL STATE WITH $\Xi_{c}^{0}$}

In this section, the analysis of the final state with the $\Xi_{c}^{0}$ is described. The reconstruction of the $\Xi_{c}^{0}$ is presented first, followed by the analysis of the $\Xi_{c}(2645)^{+}$. Finally, a search for $\Xi_{c c}^{+(+)}$decaying into the $\Xi_{c}^{0} \pi^{+}\left(\pi^{+}\right)$final state is described.

\section{A. Reconstruction of $\Xi_{c}^{0}$}

The $\Xi_{c}^{0}$ is reconstructed in three decay modes: $\Xi^{-} \pi^{+}$, $\Lambda K^{-} \pi^{+}$, and $p K^{-} K^{-} \pi^{+}$. The $\Lambda$ is reconstructed from its decay into $p \pi^{-}$. The proton and $\pi^{-}$tracks for $\Lambda$ candidates are fitted to a common vertex. The fitting result is used to suppress misreconstructed $\Lambda$ candidates and to perform the subsequent vertex fit for the $\Xi^{-} \rightarrow \Lambda \pi^{-}$or $\Xi_{c}^{0} \rightarrow \Lambda K^{-} \pi^{+}$. The invariant mass of the $\Lambda$ candidate is required to be within $3 \mathrm{MeV} / c^{2}$ of the nominal $\Lambda$ mass, which corresponds to approximately $3 \sigma$ of the mass resolution. The selection based on their decay vertex information is also applied [48]. The $\Xi^{-}$is reconstructed from its decay into $\Lambda \pi^{-}$. The $\Lambda$ and $\pi^{-}$tracks for $\Xi^{-}$ candidates are fitted to a common vertex. The fitting result is used to clean up the $\Xi^{-}$candidates and in the common vertex fit for the $\Xi_{c}^{0} \rightarrow \Xi^{-} \pi^{+}$. The closest distance of the $\Lambda$ and $\pi^{-}$ along the $z$-direction is required to be less than $3 \mathrm{~mm}$. We require $\cos \theta>0.95$, where $\theta$ is the angle between the momentum vector of the $\Xi^{-}$and the vector between the IP and the $\Xi^{-}$decay vertex. The $\chi^{2}$ of the common-vertex fit of the $\Lambda \pi^{-}$is required to be less than 50 . The invariant mass of a $\Xi^{-}$candidate is required to be within $4 \mathrm{MeV} / c^{2}$ of the nominal $\Xi^{-}$mass, which corresponds to approximately $3 \sigma$ of the mass resolution. The daughter particles of the $\Xi_{c}^{0}$ are fitted to a common vertex. The $\Xi_{c}^{0}$ candidates are selected by requiring invariant masses of the daughter particles with common vertex fit to be within 12,7 , and $7 \mathrm{MeV} / c^{2}$ of the nominal $\Xi_{c}^{0}$ mass for the $\Xi^{-} \pi^{+}, \Lambda K^{-} \pi^{+}$, and $p K^{-} K^{-} \pi^{+}$ decay modes, respectively, which correspond to approximately $1.5 \sigma$ of the mass resolution. The $\chi^{2}$ value of the common vertex fit for the $\Xi_{c}^{0}$ is required to be less than 50 . The mass constraint fit to the $\Xi_{c}^{0}$ mass is performed.

We optimize the selection criteria for $x_{p}$ in the $\Xi_{c}^{0} \pi^{+}\left(\pi^{+}\right)$ system with the method described in Sec. III B, again assuming that the $x_{p}$ spectrum for the $\Xi_{c c}$ is the same as that for the $\Lambda_{c}^{+}$. We require $0.45<x_{p}<1.0$ independent of the $\Xi_{c c}$ mass and the $\Xi_{c}^{0}$ decay mode. The same cut is applied for the analysis of the $\Xi_{c}(2645)^{+}$.

\section{B. Study of the $\Xi_{c}^{+}(\mathbf{2 6 4 5})$}

Unlike the $\Lambda_{c}^{+}$study, the signal-to-background ratio of the $\Xi_{c}^{0}$ largely depends on the decay modes. Therefore, to improve our sensitivity for the $\Xi_{c c}$, we perform a simultaneous fit to the mass spectra for the three $\Xi_{c}^{0}$ decays with fixed relative signal ratios. We use the relative yields of the $\Xi_{c}(2645)^{+} \rightarrow \Xi_{c}^{0} \pi^{+}$measured for the $\Xi_{c}^{0}$ decay modes to estimate a relative signal yield of the $\Xi_{c c}$. The relative signal yields of the $\Xi_{c c}\left(N_{\Xi_{c c}}^{i}\right)$ in a given $\Xi_{c}^{0}$ decay channel can be written as

$$
N_{\Xi_{c c}}^{i}=N_{\Xi_{c}(2645)^{+}}^{i} \frac{\epsilon_{\Xi_{c c}}^{i}}{\epsilon_{\Xi_{c}(2645)^{+}}^{i}},
$$

where $N_{\Xi_{c}(2645)^{+}}^{i}$ is the $\Xi_{c}(2645)^{+}$yield, $\epsilon_{\Xi_{c c}}^{i}$ is the reconstruction efficiency of the $\Xi_{c c}$, and $\epsilon_{\Xi_{c}}^{i}(2645)^{+}$is the reconstruction efficiency of the $\Xi_{c}(2645)^{+}$. Both efficiencies include the secondary branching fractions for $\Lambda \rightarrow p \pi^{-}$of $(63.9 \pm 0.5) \%$ and $\Xi^{-} \rightarrow \Lambda \pi^{-}$of $(99.887 \pm 0.035) \%$. The index $i$ denotes the decay mode of the $\Xi_{c}^{0}$.

Figure 4 shows the $M\left(\Xi_{c}^{0} \pi^{+}\right)$distribution for each $\Xi_{c}^{0}$ decay mode below the $\Xi_{c c}$ search region. Clear peaks corresponding to the $\Xi_{c}(2645)^{+}$are seen in all decay modes. The bump structures near $2.68 \mathrm{GeV} / c^{2}$ originate from the process $\Xi_{c}(2790)^{+} \rightarrow \Xi_{c}{ }^{\prime 0} \pi^{+} \rightarrow \Xi_{c}^{0} \gamma \pi^{+}$with a $\gamma$ missing in the reconstruction. The simultaneous UML fit is applied to extract the relative yields and the width of the $\Xi_{c}(2645)^{+}$. The $\Xi_{c}(2645)^{+}$signal is represented by a
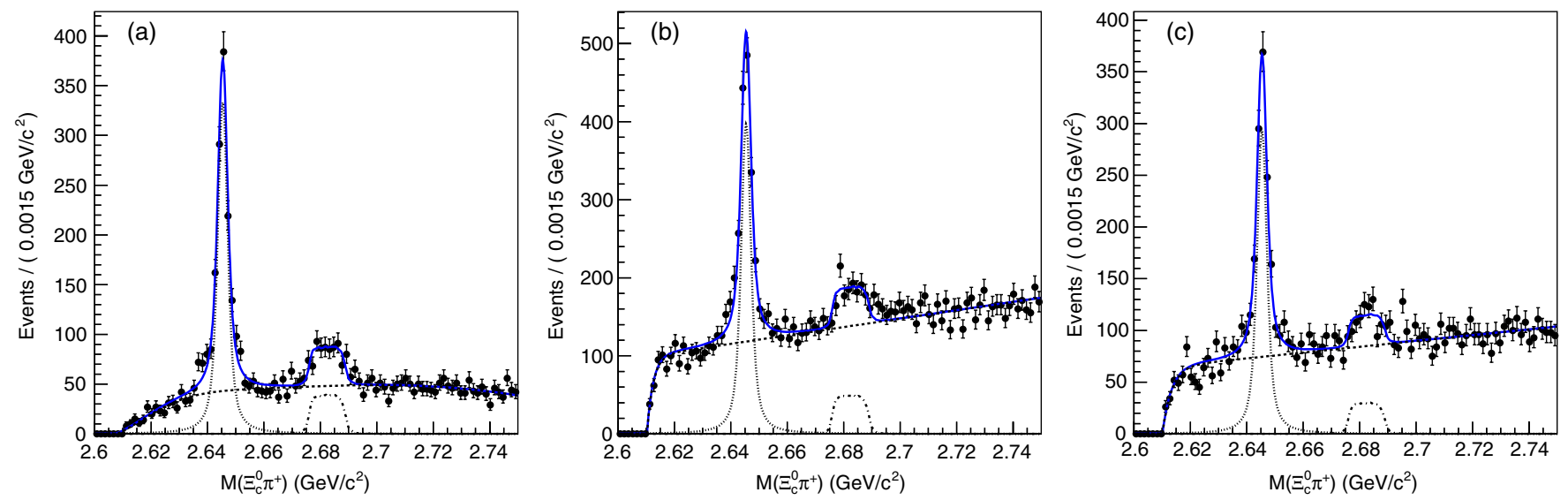

FIG. 4 (color online). $\quad M\left(\Xi_{c}^{0} \pi^{+}\right.$) distributions below the $\Xi_{c c}$ search region for (a) $\Xi_{c}^{0} \rightarrow \Xi^{-} \pi^{+}$, (b) $\Xi_{c}^{0} \rightarrow \Lambda K^{-} \pi^{+}$, (c) $\Xi_{c}^{0} \rightarrow p K^{-} K^{-} \pi^{+}$. The solid lines show the fit result. The dashed, dotted, and dash-dotted lines show the contributions from background, $\Xi_{c}(2645)^{+}$, and $\Xi_{c}(2790)^{+}$, respectively. 
Breit-Wigner function convolved with a Gaussian whose width corresponds to the mass resolution $\sigma_{\text {res }}$. The value of $\sigma_{\text {res }}$ is $1.05 \mathrm{MeV} / c^{2}$, independent of the decay modes of the $\Xi_{c}^{0}$. The PDF of the $\Xi_{c}(2790)^{+}$reflection is modeled using MC. $f_{2}(x)$ in Eq. (2) is used as the background PDF for the $\Xi_{c}^{0} \rightarrow \Xi^{-} \pi^{+}$decay mode whereas $f_{1}(x)$ in Eq. (1) is used for the $\Xi_{c}^{0} \rightarrow \Lambda K^{-} \pi^{+}$and $\Xi_{c}^{0} \rightarrow p K^{-} K^{-} \pi^{+}$decay modes. The width and mass of the $\Xi_{c}(2645)^{+}$are constrained to be the same for the three decay modes. The yield of the $\Xi_{c}(2645)^{+}$is $1298 \pm 51,1444 \pm 58$, and $974 \pm 47$ for the $\Xi^{-} \pi^{+}, \Lambda K^{-} \pi^{+}$, and $p K^{-} K^{-} \pi^{+}$decay mode, respectively. The mass and width are obtained to be $2645.4 \pm 0.1 \mathrm{MeV} / c^{2}$ and $2.6 \pm 0.2 \mathrm{MeV} / c^{2}$, respectively. The $\chi^{2} / n d f$ of the fit for the binning in Fig. 4 is 296/276.

To check the consistency of the width measurement between the $\Xi_{c}^{0}$ decay modes, we fit the three mass spectra separately. The measured widths are found to be consistent between the three decay modes: $2.9 \pm 0.3 \mathrm{MeV} / c^{2}$, $2.6 \pm 0.3 \mathrm{MeV} / c^{2}$, and $2.5 \pm 0.3 \mathrm{MeV} / c^{2}$ for $\Xi_{c}^{0} \rightarrow \Xi^{-} \pi^{+}$, $\Lambda K^{-} \pi^{+}$, and $p K^{-} K^{-} \pi^{+}$, respectively. The measured width is found to be consistent for the three decay modes. The systematic uncertainty of the width measurement due to the fit procedure is studied with pseudoexperiment events samples: the $\Xi_{c}(2645)^{+}$component is generated according to the signal $\mathrm{MC}$ sample with the natural width of $2.6 \mathrm{MeV} / c^{2}$ by signal $\mathrm{MC}$, while contributions from background and $\Xi_{c}(2790)^{+}$reflection are generated based on the fit result with the real data. The statistics of the pseudoexperiment samples are the same as for those of data. The width of the $\Xi_{c}(2645)^{+}$is extracted from simultaneous fits to pseudoexperiment samples, and its mean value is obtained to be $2.75 \pm 0.03 \mathrm{MeV} / c^{2}$, which is higher than the input value by $0.15 \mathrm{MeV} / c^{2}$. The difference is included as the systematic uncertainty from the fit procedure. The systematic uncertainty due to the background shape is studied by fitting the data with a second-order polynomial function for the alternative background shape. The fit region is restricted to $2.62-2.75 \mathrm{GeV} / c^{2}$. The width is obtained to be $2.9 \pm 0.2 \mathrm{MeV} / c^{2}$, which is $0.3 \mathrm{MeV} / c^{2}$ higher than the default measurement. This deviation is included as a systematic uncertainty. To check the systematic uncertainty due to the mass resolution, we evaluate the ratio of the resolution of the $\Xi_{c}^{0}$ in the data and $\mathrm{MC}, \frac{\sigma_{\text {data }}}{\sigma_{\mathrm{mc}}}$, where $\sigma_{\text {data }}$ is the resolution of $\Xi_{c}^{0}$ for data and $\sigma_{\mathrm{mc}}$ is that for MC. An additional cut of $2.64 \mathrm{GeV} / c^{2}<M\left(\Xi_{c}^{0} \pi^{+}\right)<2.65 \mathrm{GeV} / c^{2}$ is applied to select the $\Xi_{c}(2645)^{+}$region only. $\frac{\sigma_{\text {data }}}{\sigma_{\mathrm{mc}}}$ is $0.96 \pm 0.03,1.03 \pm 0.07$, and $1.07 \pm 0.03$ for the $\Xi^{-} \pi^{+}$, $\Lambda K^{-} \pi^{+}$, and $p K^{-} K^{-} \pi^{+}$decay modes, respectively. We assign the systematic uncertainty conservatively by increasing the mass resolution by $7 \%$, which is the largest deviation of the $\frac{\sigma_{\text {data }}}{\sigma_{\mathrm{mc}}}$ from unity, for all the decay modes. The resulting width is $2.5 \pm 0.2 \mathrm{MeV} / c^{2}$. The difference of $0.1 \mathrm{MeV} / c^{2}$ with respect to the default measurement is included as a systematic uncertainty. We use alternative $x_{p}$ ranges of $0.35<x_{p}<1.0$ and $0.55<x_{p}<1.0$ and extract the width of $2.6 \pm 0.2 \mathrm{MeV} / c^{2}$ for the former case and $2.8 \pm$ $0.2 \mathrm{MeV} / c^{2}$ for the latter. The largest difference of $0.2 \mathrm{MeV} / c^{2}$ with respect to the default measurement is included as a systematic uncertainty. By adding all the systematic uncertainties in quadrature, the total systematic uncertainty for the width measurement is estimated to be $0.4 \mathrm{MeV} / c^{2}$.

\section{Search for doubly charmed baryons in the $\Xi_{c}^{\mathbf{0}} \boldsymbol{\pi}^{+}\left(\boldsymbol{\pi}^{+}\right)$final state}

To obtain the relative yields of the $\Xi_{c c}, \varepsilon_{\Xi_{c c}}^{i}$ and $\varepsilon_{\Xi_{c}\left(2645^{+}\right)}^{i}$ are evaluated using MC, and the efficiency $\varepsilon_{\Xi_{c c}}^{i}$ is obtained as a function of the $\Xi_{c c}$ mass. $N_{\Xi_{c}(2645)^{+}}^{i}$ and $\varepsilon_{\Xi_{c}\left(2645^{+}\right)}^{i}$ are summarized in Table IV, while $\varepsilon_{\Xi_{c c}^{+}}^{i}$ as a function of $\Xi_{c c}$ mass is shown in Fig. 5. As an example, the relative yield ratio of the $\Xi_{c c}^{+}$with a mass of $3.6 \mathrm{GeV} / c^{2}$ is $N \Xi_{\Xi_{c c}}^{\Xi^{-}} \pi^{+}: N_{\Xi_{c c}}^{\Lambda K^{-} \pi^{+}}: N_{\Xi_{c c}}^{p K^{-} K^{-} \pi^{+}}=1: 1.15: 0.84$.

Figures 6 and 7 (a) $-(\mathrm{c})$ show the $M\left(\Xi_{c}^{0} \pi^{+}\left(\pi^{+}\right)\right)$distribution in the $\Xi_{c c}$ search region with all the selection cuts applied, overlaid with the MC expectation for the $\Xi_{c c}$ at the mass of $3.60 \mathrm{GeV} / c^{2}$ with $\sigma\left(e^{+} e^{-} \rightarrow \Xi_{c c}^{+(+)} X\right)$ of $500 \mathrm{fb}$ and both $\mathcal{B}\left(\Xi_{c c}^{+(+)} \rightarrow \Xi_{c}^{0} \pi^{+}\left(\pi^{+}\right)\right)$and $\mathcal{B}\left(\Xi_{c}^{0} \rightarrow \Xi^{-} \pi^{+}\right)$of $5 \%$. The relative yields of signal MC for each decay mode are based on $N_{\Xi_{c c}}^{i}$.

A simultaneous UML fit, with the relative $\Xi_{c c}$ yields constrained as discussed earlier, is applied to evaluate the statistical significance of the $\Xi_{c c}$. The signal PDF is described with MC events generated for each decay mode and with the $\Xi_{c c}$ mass generated in the search region with a $1 \mathrm{MeV} / c^{2}$ step. The mass resolution is $2.7-4.2 \mathrm{MeV} / c^{2}$, depending on the mass of the $\Xi_{c c}$. The background PDF is modeled as a third-order polynomial. The highest significance is $3.2 \sigma$ for the mass around $3.553 \mathrm{GeV} / c^{2}$ in the $M\left(\Xi_{c}^{0} \pi^{+}\right)$. We perform a pseudoexperiment test to evaluate the probability of observing a peak with such a statistical significance. A smooth mass distribution based on data is generated and the significance is evaluated in the entire search region. The probability to observe a peak with a significance higher than $3.2 \sigma$ in one pseudoexperiment is $26 \%$. Therefore, the statistical significance of $3.2 \sigma$ is insufficient to claim evidence of the $\Xi_{c c}^{+}$.

TABLE IV. $\Xi_{c}(2645)^{+}$yields and efficiencies used for estimation of the relative $\Xi_{c c}$ yields.

\begin{tabular}{lrc}
\hline \hline Decay mode & $N_{\Xi_{c}(2645)^{+}}$ & $\varepsilon_{\Xi_{c}(2645)^{+}}$ \\
\hline$\Xi^{-} \pi^{+}$ & $1298 \pm 51$ & $0.0748 \pm 0.0002$ \\
$\Lambda K^{-} \pi^{+}$ & $1444 \pm 58$ & $0.0977 \pm 0.0003$ \\
$p K^{-} K^{-} \pi^{+}$ & $974 \pm 47$ & $0.1920 \pm 0.0005$ \\
\hline \hline
\end{tabular}



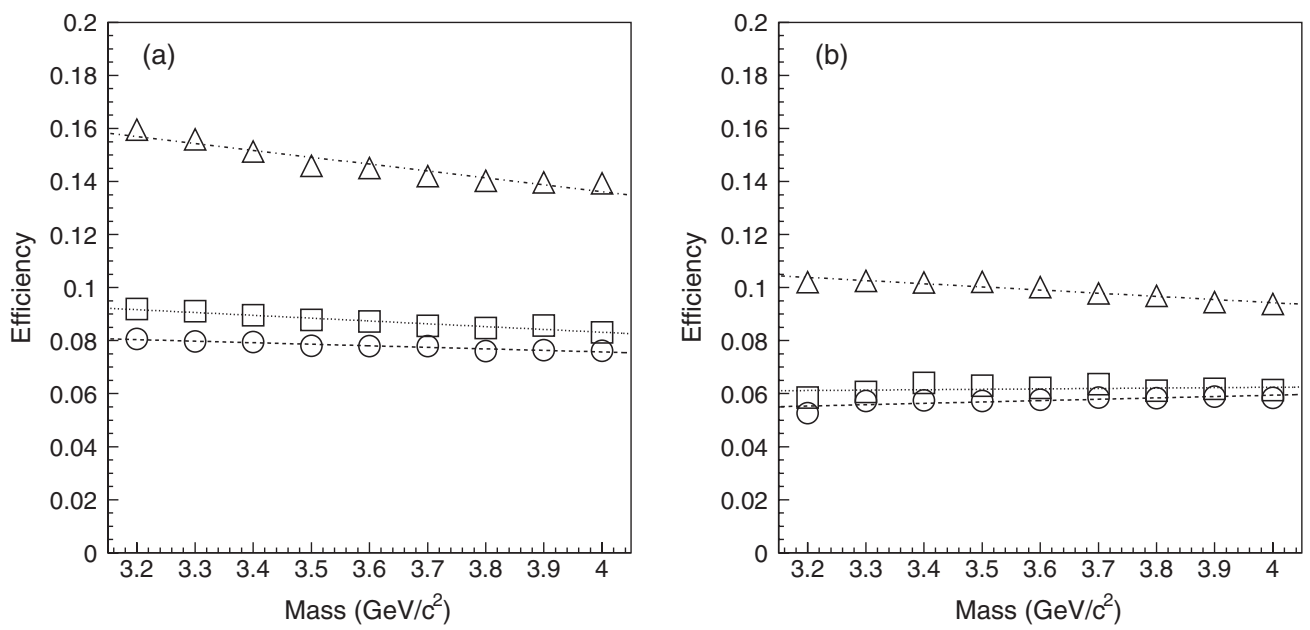

FIG. 5. Reconstruction efficiencies for the $\Xi_{c c}$ as a function of the $\Xi_{c c}$ mass for (a) $\Xi_{c c}^{+}$, (b) $\Xi_{c c}^{++}$. Circles, square, and triangle points are for $\Xi_{c}^{0} \rightarrow \Xi^{-} \pi^{+}, \Lambda K^{-} \pi^{+}$, and $p K^{-} K^{-} \pi^{+}$, respectively. The lines are the result of the fit with a linear function.
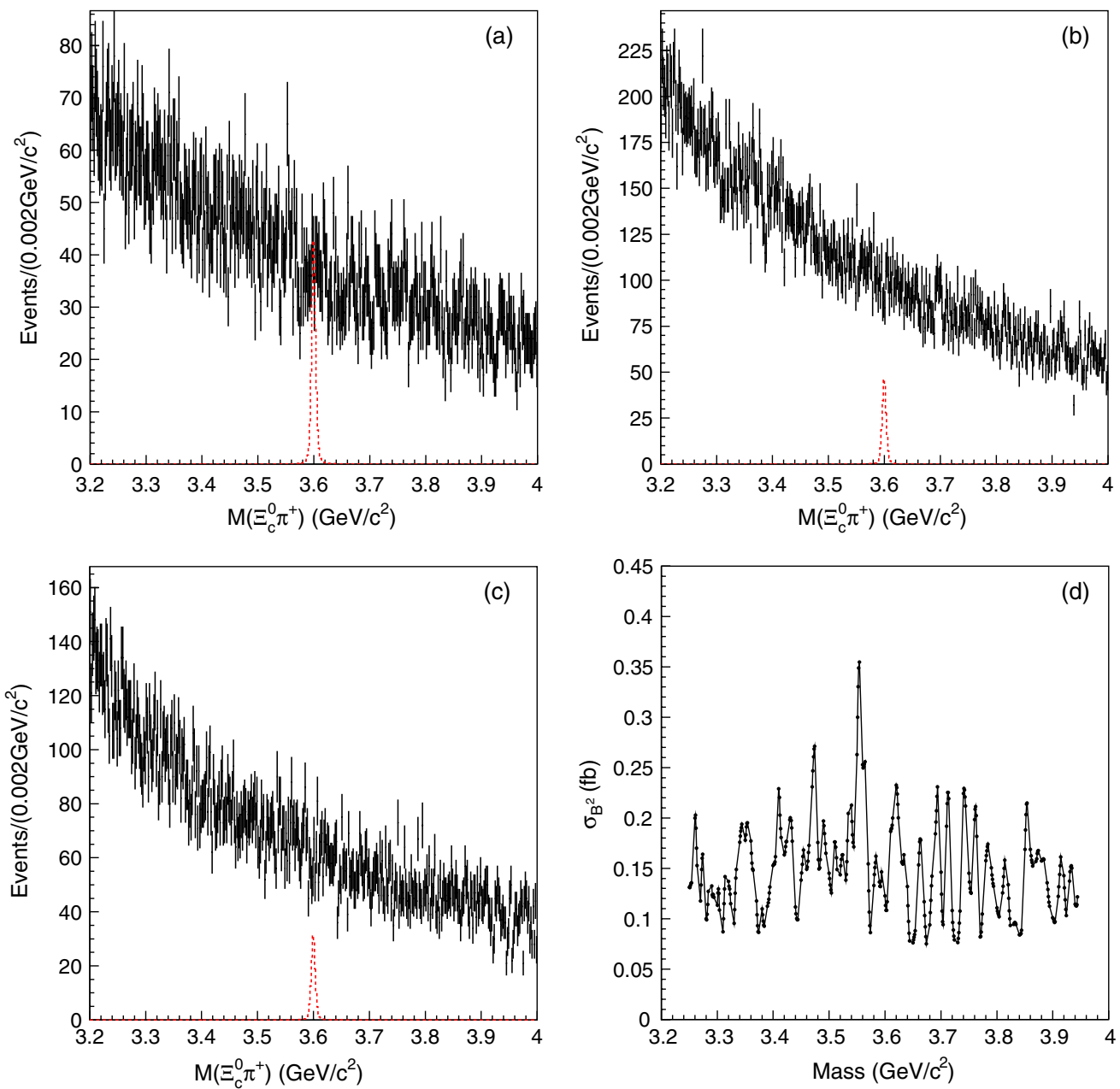

FIG. 6 (color online). (a)-(c): $M\left(\Xi_{c}^{0} \pi^{+}\right)$distribution in the $\Xi_{c c}^{+}$search region for $\Xi_{c}^{0} \rightarrow$ (a) $\Xi^{-} \pi^{+}$, (b) $\Lambda K^{-} \pi^{+}$, (c) $p K^{-} K^{-} \pi^{+}$. The vertical error bars are from data. The dashed histograms are from signal MC. (d): 95\% C.L. upper limit of the $\sigma_{\mathcal{B}^{2}}$ for $\Xi_{c c}^{+}$as a function of the mass with a $1 \mathrm{MeV} / c^{2}$ step. 

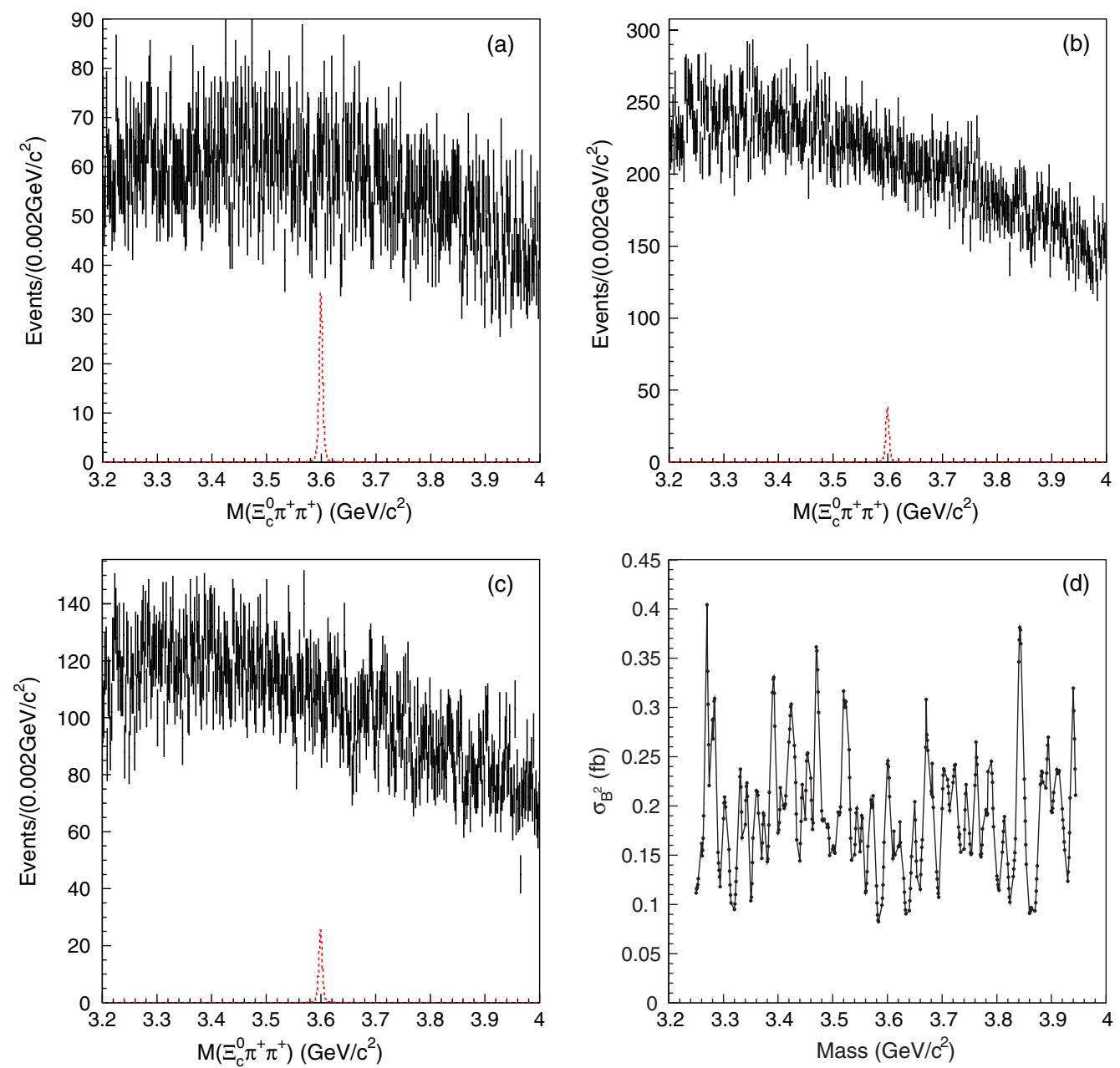

FIG. 7 (color online). (a)-(c): $M\left(\Xi_{c}^{0} \pi^{+} \pi^{+}\right)$distribution in the $\Xi_{c c}^{++}$search region for $\Xi_{c}^{0} \rightarrow$ (a) $\Xi^{-} \pi^{+}$, (b) $\Lambda K^{-} \pi^{+}$, (c) $p K^{-} K^{-} \pi^{+}$. The vertical error bars are from data. The dashed histograms are from signal MC. (d): 95\% C.L. upper limit of the $\sigma_{\mathcal{B}^{2}}$ for $\Xi_{c c}^{++}$as a function of the mass with a $1 \mathrm{MeV} / \mathrm{c}^{2}$ step.

The $95 \%$ C.L. upper limit for the product of the cross section and branching fractions produced with $0.45<x_{p}<1.0$ condition,

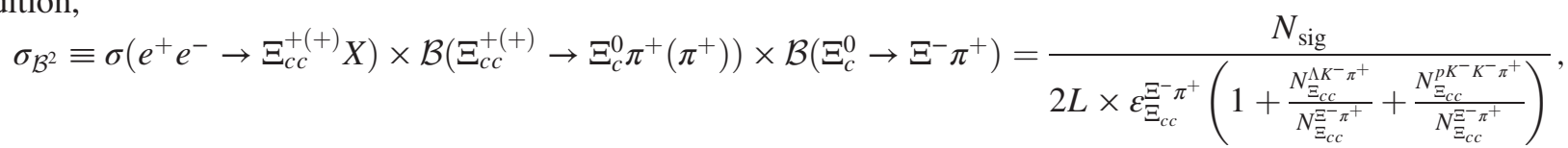

is evaluated with the same method as in Sec. III B. In addition to the sources from the study with the $\Lambda_{c}^{+}$, two others are included here. The systematic uncertainty from the $\Lambda$ reconstruction efficiency is estimated to be $3 \%$ using the yield of the $B^{+} \rightarrow \Lambda \bar{\Lambda} K^{+}$with and without the requirement using decay vertex information. The systematic uncertainties related to $N_{\Xi_{c}(2645)^{+}}^{i}$ are taken from their statistical errors. The systematic uncertainties are summarized in the fourth column in Table II. Figures 6 and 7(d) show $\sigma_{\mathcal{B}^{2}}$ for the $\Xi_{c c}^{+(+)}$as a function of the mass with a $1 \mathrm{MeV} / c^{2}$ step. The $95 \%$ C.L. upper limit on $\sigma_{\mathcal{B}^{2}}$ is $0.076-$ $0.35 \mathrm{fb}$ for the $\Xi_{c c}^{+}$and $0.082-0.40 \mathrm{fb}$ for the $\Xi_{c c}^{++}$.

\section{CONCLUSION}

We have presented a search for doubly charmed baryons and a study of the charmed strange baryons $\Xi_{c}(3055)^{+}$, $\Xi_{c}(3123)^{+}$and $\Xi_{c}(2645)^{+}$using the full data sample $\left(980 \mathrm{fb}^{-1}\right)$ collected with the Belle detector. The search for doubly charmed baryons is an improved study of our previous work [2]. We use about two times statistics of previous work and several additional decay modes that were not studied in the previous work.

We search for the $\Xi_{c c}$ in the $\Lambda_{c}^{+} K^{-} \pi^{+}\left(\pi^{+}\right)$and $\Xi_{c}^{0} \pi^{+}\left(\pi^{+}\right)$final states. The $\Lambda_{c}^{+}$is reconstructed from the $p K^{-} \pi^{+}$and $p K_{S}^{0}$ decay modes. We do not find any significant $\Xi_{c c}$ signal and set a 95\% C.L. upper limit on 
$\sigma\left(e^{+} e^{-} \rightarrow \Xi_{c c}^{+(+)} X\right) \times \mathcal{B}\left(\Xi_{c c}^{+(+)} \rightarrow \Lambda_{c}^{+} K^{-} \pi^{+}\left(\pi^{+}\right)\right)$with the scaled momentum $0.5<x_{p}<1.0: 4.1-25.0 \mathrm{fb}$ for $\Xi_{c c}^{+}$and 2.5-26.5 fb for $\Xi_{c c}^{++}$. We also search for the $\Xi_{c c}$ in the $\Xi_{c}^{0} \pi^{+}\left(\pi^{+}\right)$final state. The $\Xi_{c}^{0}$ is reconstructed from the $\Xi^{-} \pi^{+}, \Lambda K^{-} \pi^{+}$, and $p K^{-} K^{-} \pi^{+}$decay modes. We do not find any significant $\Xi_{c c}$ signal and set a $95 \%$ C.L. upper limit on $\sigma\left(e^{+} e^{-} \rightarrow \Xi_{c c}^{+(+)} X\right) \times \mathcal{B}\left(\Xi_{c c}^{+(+)} \rightarrow \Xi_{c}^{0} \pi^{+}\left(\pi^{+}\right)\right) \times$ $\mathcal{B}\left(\Xi_{c}^{0} \rightarrow \Xi^{-} \pi^{+}\right)$with the scaled momentum $0.45<$ $x_{p}<1.0$ : $0.076-0.35 \mathrm{fb}$ for the $\Xi_{c c}^{+}$and $0.082-0.40 \mathrm{fb}$ for the $\Xi_{c c}^{++}$. When we compare these values with the measurements by $B A B A R$, we should note several things. We should multiply our result by two as written in Sec. III. For the final states with $\Lambda_{c}^{+}$, their upper limit is for the product of the cross section, $\mathcal{B}\left(\Xi_{c c}^{+(+)} \rightarrow \Lambda_{c}^{+} K^{-} \pi^{+}\left(\pi^{+}\right)\right)$ and $\mathcal{B}\left(\Lambda_{c}^{+} \rightarrow p K^{-} \pi^{+}\right)$. The values presented in Ref. [34] are the highest upper limits in the search region, which can be compared with our highest values. After taking into account these points, we find our limits represent improvements by about a factor two for the final states with $\Lambda_{c}^{+}$and a factor of four for the final states with $\Xi_{c}^{0}$.

If we assume $\mathcal{B}\left(\Xi_{c c}^{+(+)} \rightarrow \Lambda_{c}^{+} K^{-} \pi^{+}\left(\pi^{+}\right)\right), \mathcal{B}\left(\Xi_{c c}^{+(+)} \rightarrow\right.$ $\left.\Xi_{c}^{0} \pi^{+}\left(\pi^{+}\right)\right)$, and $\mathcal{B}\left(\Xi_{c}^{0} \rightarrow \Xi^{-} \pi^{+}\right)$to be $5 \%$, which is equal to the $\mathcal{B}\left(\Lambda_{c}^{+} \rightarrow p K^{-} \pi^{+}\right)$, the upper limits on the $\sigma\left(e^{+} e^{-} \rightarrow\right.$ $\left.\Xi_{c c}^{+} X\right)$ are $82-500 \mathrm{fb}\left(\Xi_{c c}^{+}\right)$and $50-530 \mathrm{fb}\left(\Xi_{c c}^{++}\right)$for the decay mode with the $\Lambda_{c}^{+}$and 30-140 $\mathrm{fb}\left(\Xi_{c c}^{+}\right)$and 33-160 fb $\left(\Xi_{c c}^{++}\right)$for the decay mode with the $\Xi_{c}^{0}$. These values are comparable to some of the theoretical predictions [28,29].

We have searched for the $\Xi_{c}(3055)^{+}$and $\Xi_{c}(3123)^{+}$ in the $\Lambda_{c}^{+} K^{-} \pi^{+}$decays through intermediate $\Sigma_{c}(2455)^{++}$ or $\Sigma_{c}(2520)^{++}$states. We observe the $\Xi_{c}(3055)^{+}$with a significance of $6.6 \sigma$, including systematic uncertainty. The mass and width are measured to be $3058.1 \pm 1.0$ (stat) \pm 2.1 (sys) $\mathrm{MeV} / c^{2}$ and $9.7 \pm 3.4$ (stat) \pm 3.3 (sys) $\mathrm{MeV} / c^{2}$, respectively. We do not observe any significant signal corresponding to the $\Xi_{c}(3123)^{+}$.

The first measurement of the width of the $\Xi_{c}(2645)^{+}$ has been also performed, yielding $2.6 \pm 0.2$ (stat) \pm 0.4 (sys) $\mathrm{MeV} / c^{2}$.

\section{ACKNOWLEDGMENTS}

We thank the KEKB group for the excellent operation of the accelerator; the KEK cryogenics group for the efficient operation of the solenoid; and the KEK computer group, the National Institute of Informatics, and the PNNL/EMSL computing group for valuable computing and SINET4 network support. We acknowledge support from the Ministry of Education, Culture, Sports, Science, and Technology (MEXT) of Japan, the Japan Society for the Promotion of Science (JSPS), and the Tau-Lepton Physics Research Center of Nagoya University; the Australian Research Council and the Australian Department of Industry, Innovation, Science and Research; Austrian Science Fund under Grant No. P 22742-N16; the National Natural Science Foundation of China under Contracts No. 10575109, No. 10775142, No. 10825524 , No. 10875115, No. 10935008, and No. 11175187; the Ministry of Education, Youth and Sports of the Czech Republic under Contract No. MSM0021620859; the Carl Zeiss Foundation, the Deutsche Forschungsgemeinschaft and the VolkswagenStiftung; the Department of Science and Technology of India; the Istituto Nazionale di Fisica Nucleare of Italy; The WCU program of the Ministry Education Science and Technology, National Research Foundation of Korea Grants No. 20110029457, No. 2012-0008143, No. 2012R1A1A2008330, No. 2013R1A1A3007772, BRL program under NRF Grant No. KRF-2011-0020333, BK21 Plus program, and GSDC of the Korea Institute of Science and Technology Information; the Polish Ministry of Science and Higher Education and the National Science Center; the Ministry of Education and Science of the Russian Federation and the Russian Federal Agency for Atomic Energy; the Slovenian Research Agency; the Basque Foundation for Science (IKERBASQUE) and the UPV/EHU under program UFI 11/55; the Swiss National Science Foundation; the National Science Council and the Ministry of Education of Taiwan; and the U.S. Department of Energy and the National Science Foundation. This work is supported by a Grant-in-Aid from MEXT for Science Research in a Priority Area ("New Development of Flavor Physics"), from JSPS for Creative Scientific Research ("Evolution of Tau-lepton Physics"), and Grant-in-Aid for Scientific Research on Innovative Areas "Elucidation of New Hadrons with a Variety of Flavors".
[1] R. Mizuk et al. (Belle Collaboration), Phys. Rev. Lett. 94, 122002 (2005).

[2] R. Chistov et al. (Belle Collaboration), Phys. Rev. Lett. 97, 162001 (2006).

[3] B. Aubert et al. (BABAR Collaboration), Phys. Rev. Lett. 97, 232001 (2006).
[4] B. Aubert et al. (BABAR Collaboration), Phys. Rev. Lett. 98, 012001 (2007).

[5] K. Abe et al. (Belle Collaboration), Phys. Rev. Lett. 98, 262001 (2007).

[6] B. Aubert et al. (BABAR Collaboration), Phys. Rev. D 77, 012002 (2008). 
[7] E. Solovieva, R. Chistov, I. Adachi, H. Aihara, K. Arinstein, T. Aushev, A. M. Bakich, and V. Balagura et al., Phys. Lett. B 672, 1 (2009).

[8] T. Lesiak et al. (Belle Collaboration), Phys. Lett. B 665, 9 (2008).

[9] J. Beringer et al. (Particle Data Group Collaboration), Phys. Rev. D 86, 010001 (2012).

[10] R. Roncaglia, D. B. Lichtenberg, and E. Predazzi, Phys. Rev. D 52, 1722 (1995).

[11] D. Ebert, R. N. Faustov, V. O. Galkin, A. P. Martynenko, and V. A. Saleev, Z. Phys. C 76, 111 (1997).

[12] B. Silvestre-Brac, Prog. Part. Nucl. Phys. 36, 263 (1996).

[13] S.-P. Tong, Y.-B. Ding, X.-H. Guo, H.-Y. Jin, X.-Q. Li, P.-N. Shen, and R. Zhang, Phys. Rev. D 62, 054024 (2000).

[14] S. M. Gerasyuta and D. V. Ivanov, Nuovo Cimento A 112, 261 (1999).

[15] C. Itoh, T. Minamikawa, K. Miura, and T. Watanabe, Phys. Rev. D 61, 057502 (2000).

[16] V. V. Kiselev and A. K. Likhoded, Usp. Fiz. Nauk 172, 497 (2002) [Phys. Usp. 45, 455 (2002)].

[17] I. M. Narodetskii and M. A. Trusov, arXiv:hep-ph/0204320.

[18] D. Ebert, R. N. Faustov, V. O. Galkin, and A. P. Martynenko, Phys. Rev. D 66, 014008 (2002).

[19] J. Vijande, H. Garcilazo, A. Valcarce, and F. Fernandez, Phys. Rev. D 70, 054022 (2004).

[20] S. Migura, D. Merten, B. Metsch, and H.-R. Petry, Eur. Phys. J. A 28, 41 (2006).

[21] C. Albertus, E. Hernandez, J. Nieves, and J. M. VerdeVelasco, Eur. Phys. J. A 32, 183 (2007); C. Albertus, E. Hernandez, J. Nieves, and J. M. Verde-Velasco, Eur. Phys. J. A 36, 119(E) (2008).

[22] W. Roberts and M. Pervin, Int. J. Mod. Phys. A 23, 2817 (2008).

[23] R. Lewis, N. Mathur, and R. M. Woloshyn, Phys. Rev. D 64, 094509 (2001).

[24] H. Na and S. Gottlieb, Proc. Sci., LATTICE (2008) 119 [arXiv:0812.1235].

[25] L. Liu, H.-W. Lin, K. Orginos, and A. Walker-Loud, Phys. Rev. D 81, 094505 (2010).

[26] Y. Namekawa, (PACS-CS Collaboration) Proc. Sci., LATTICE (2012) 139 [arXiv:1212.0073].

[27] C. Alexandrou, J. Carbonell, D. Christaras, V. Drach, M. Gravina, and M. Papinutto, Phys. Rev. D 86, 114501 (2012).
[28] V. V. Kiselev, A. K. Likhoded, and M. V. Shevlyagin, Phys. Lett. B 332, 411 (1994).

[29] J. P. Ma and Z. G. Si, Phys. Lett. B 568, 135 (2003).

[30] V. V. Braguta, V. V. Kiselev, and A. E. Chalov, Yad. Fiz. 65, 1575 (2002) [Phys. At. Nucl. 651537 (2002)].

[31] M. Mattson et al. (SELEX Collaboration), Phys. Rev. Lett. 89, 112001 (2002).

[32] A. Ocherashvili et al. (SELEX Collaboration), Phys. Lett. B 628, 18 (2005).

[33] S. P. Ratti, Nucl. Phys. B, Proc. Suppl. 115, 33 (2003).

[34] B. Aubert et al. (BABAR Collaboration), Phys. Rev. D 74, 011103 (2006).

[35] R. Aaij et al. (LHCb Collaboration), J. High Energy Phys. 12 (2013) 090.

[36] L. Gibbons et al. (CLEO Collaboration), Phys. Rev. Lett. 77, 810 (1996).

[37] S. Kurokawa and E. Kikutani, Nucl. Instrum. Methods Phys. Res., Sect. A 499, 1 (2003), and other papers included in this volume; T. Abe et al., Prog. Theor. Exp. Phys. (2013) 03A001 and following articles up to 03A011.

[38] A. Abashian et al. (Belle Collaboration), Nucl. Instrum. Methods Phys. Res., Sect. A 479, 117 (2002); also see detector section in J. Brodzicka et al., Prog. Theor. Exp. Phys. (2012), 04D001.

[39] Z.Natkaniec et al. (Belle SVD2 Group), Nucl. Instrum. Methods Phys. Res., Sect. A 560, 1 (2006).

[40] K. Hanagaki, H. Kakuno, H. Ikeda, T. Iijima, and T. Tsukamoto, Nucl. Instrum. Methods Phys. Res., Sect. A 485, 490 (2002).

[41] D. J. Lange, Nucl. Instrum. Methods Phys. Res., Sect. A 462, 152 (2001).

[42] T. Sjostrand, Comput. Phys. Commun. 82, 74 (1994).

[43] E. Barberio and Z. Was, Comput. Phys. Commun. 79, 291 (1994).

[44] R. Brun et al., GEANT3.21, CERN Report No. DD/EE/84-1, 1984.

[45] Throughout this paper, the inclusion of the charge-conjugate decay mode is implied unless otherwise stated.

[46] K. Sumisawa et al. (Belle Collaboration), Phys. Rev. Lett. 95, 061801 (2005).

[47] B. Aubert et al. (BABAR Collaboration), Phys. Rev. D 75, 012003 (2007).

[48] K. Abe et al. (Belle Collaboration), Phys. Rev. D 65, 091103 (2002). 